Supporting Information for

\title{
Study on Structure, Stability and Phase Transformation of Dufulin polymorphs
}

Mengnan Li, ${ }^{\dagger}$ Chengyu Gong, ${ }^{\dagger}$ Guobin Ren, ${ }^{*}$ Xiaoyong Xu, ${ }^{\dagger}$ Minghuang Hong, ${ }^{t, *}$ Zhong Li ${ }^{*}, *$

† Shanghai Key Laboratory of Chemical Biology, School of Pharmacy, East China University of

Science and Technology, No. 130 Meilong Rd., Shanghai, 200237, PR China

$\$$ State Key Laboratory of Bioreactor Engineering; Engineering Research Centre of Pharmaceutical

Process Chemistry, Ministry of Education; Laboratory of Pharmaceutical Crystal Engineering \& Technology, School of Pharmacy, East China University of Science and Technology, No. 130

Meilong Rd., Shanghai, 200237, PR China

*Corresponding author E-mail: mhhong@ecust.edu.cn

*Corresponding author E-mail: lizhong@ecust.edu.cn 


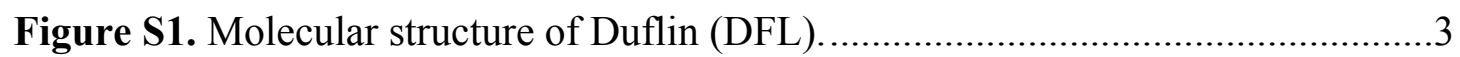

Scheme S1. Synthetic route for the preparation of the Dufulin....................................3

Table S1. The summary of selected solid-state screening experiment.........................5

Table S2. The detailed data of DFL polymorphs peaks. .........................................8

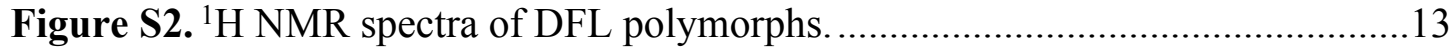

Figure S3. TGA curves for Form I-IV, VI and amorphous of DFL..........................13

Figure S4. Representative DSC and TGA curve (a) and the PXRD of desolvation process (b) for DFL Form V.

Figure S5. (a) Representative DSC curve for DFL Form VI, (b) PXRD showing the sequence of events starting with DFL Form VI.

Figure S6. (a) Representative DSC curve (green) and MDSC (blue) for DFL Amorphous, (b) PXRD showing the sequence of events starting with DFL amorphous.

Figure S7. FT-IR spectra of the various forms of DFL ...........................................16

Table S3. FT-IR spectra characteristic peaks of the various forms of DFL................16

Figure S8. The molecular structures of DFL and structurally similar molecules. ......17

Table S4. The crystal structure data of structurally similar molecules. .....................17

Figure S9. The simulated powder pattern of structurally similar molecules. .............18

Figure S10. The crystal structure of structurally similar molecules. ........................18

Figure S11. Overlay of the DFL molecules (a) orange: Form I (R), blue: Form IV (R), red: Form VI (R), (b) green: Form III (S), blue: Form IV (S), and magenta: Form V (S).

Table S5. The detailed data of torsion angles..........................................................19

Table S6. Intermolecular hydrogen bonds in Form I, III, IV, V and VI.

Figure S12. Two-dimensional fingerprint plots for DFL Forms: (a) I, (b) III, (c) IV, (d) $\mathrm{V}$ and (e) VI.

Figure S13. The PXRD patterns of DFL polymorphs in solvent-mediated suspension experiments. (a) The physically mixture of all DFL forms at different temperature, (b) 
Form I, (c) Form II, (d) Form III, (e) Form V, (f) amorphous, (g) the mixtures of Form I and III and (h) the mixtures of Form IV and VI.

Table S7. The design and results of solvent-mediated transformation experiments...27

Figure S14. The PXRD patterns of DFL polymorphs in stability experiment. (a) Form I, (b) Form II, (c) Form III, (d) Form IV, (e) Form V, (f) Form VI, and (g) amorphous.

Figure S15. (a) Overlay of DSC curves of Form I at different heating rates, (b) Polarized-light photographs show the selected temperature-induced solid-state transformations of DFL (I $\rightarrow$ II), (c) Overlay of DSC curves of Form III at different heating rates, and (d) Polarized-light photographs show the selected temperature induced solid-state transformations of DFL (III $\rightarrow$ II)

Table S8. Solubility data of DFL Forms I and III at different temperatures...............32

Figure S16. Van't Hoff plots of log solubility for DFL Forms I and III in ethanol....33 Table S9. Fitting parameters of Van't Hoff equation for DFL Form I and III............33

Figure S17. The transformation relationship of DFL polymorphs 34

Figure S18. Dissolution-time profiles of DFL Forms I, II, and III in 50\% v/v ethanol at $25^{\circ} \mathrm{C}$. 34 


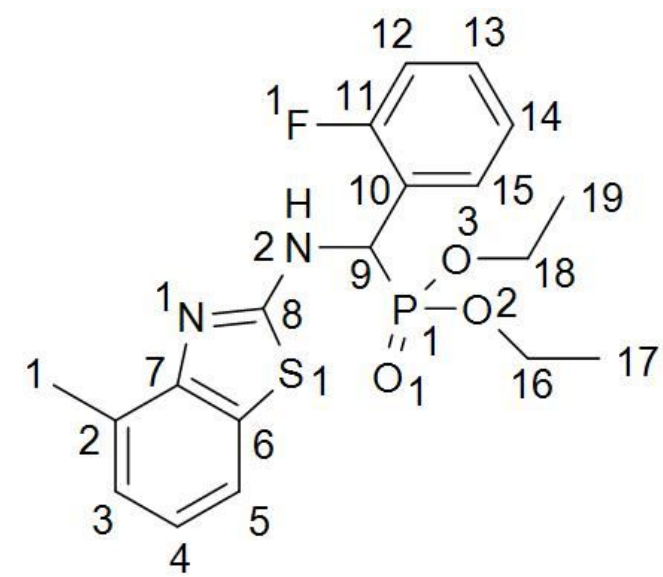

Figure S1. Molecular structure of Duflin (DFL).

The Synthesis of Dufulin. Dufulin $\left(\mathrm{C}_{19} \mathrm{H}_{22} \mathrm{FN}_{2} \mathrm{O}_{3} \mathrm{PS}\right.$, MW 408.43) was synthesized by following previously published procedures (Scheme S1). 2-amino-4methylbenzothiazole $(4 \mathrm{mmol})$ and 2-fluorobenzaldehyde $(4 \mathrm{mmol})$ was added in toluene $(15 \mathrm{~mL})$ and refluxed stirring for 7 hours to obtain the intermediate compound 1. Next, dipropyl phosphite $(4 \mathrm{mmol})$ was added to the solution and refluxed stirring for 15 hours. Finally, the solvent was removed under reduced pressure and the residue was washed with water, filtered, dried, and crystallized three times from ethanol to obtain target compound 2 (Dufulin) as colorless crystals with a yield of $83.1 \%$.

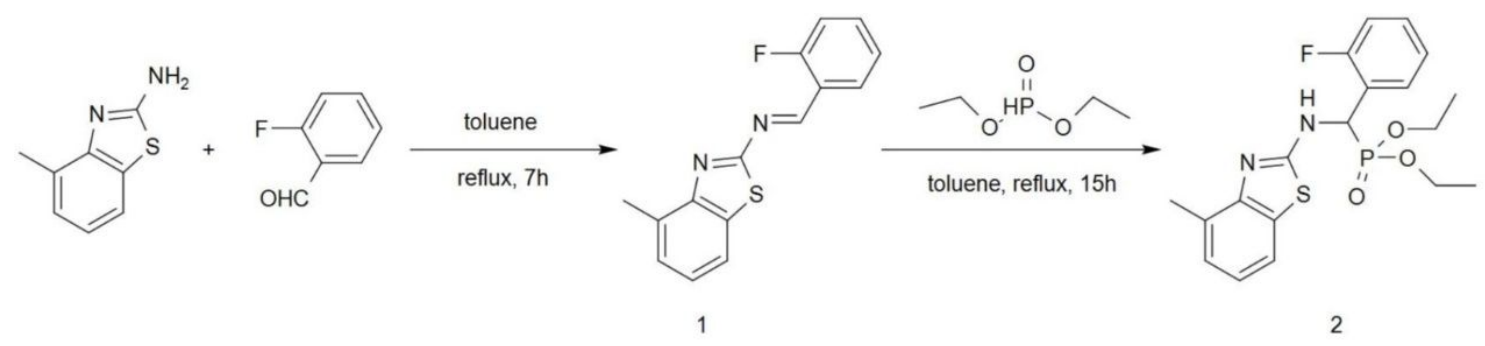

Scheme S1. Synthetic route for the preparation of the Dufulin. 
Polymorphism Screening. Comprehensive polymorphism screening had been conducted through a variety of methods including melting, cooling, evaporation, slurry and anti-solvent crystallization, etc. Sixty-eight solvents, including dichloromethane, 1,2-dichloroethane, $N, N$-dimethylformamide, tetrahydrofuran, 2,2,2-trifluoroethanol, chloroform, pyridine, acetone, ethyl acetate, 2-butanone, $N, N$-dimethylacetamide, toluene, 1,4-dioxane, ethyl formate, $N$-methylpyrrolidone, dimethyl sulfoxide, anhydrous methanol, butyl acetate, acetonitrile, 4-methyl-2-pentanone, chlorobenzene, 2-nitropropane, 3-pentanone, 2-methyltetrahydrofuran, ethylene glycol dimethyl ether, ethanol, n-propanol, iso-propanol, n-butanol, iso-butanol, sec-butanol, iso-propyl acetate, nitromethane, anhydrous ether, methyl tert-butyl ether, ethylene glycol diethyl ether, p-xylene, trifluorotoluene, cumene, mesitylene, diphenyl ether, dibenzyl ether, methyl cyclopentyl ether, anisole, phenethyl ether, 2-ethoxyethanol, m-phenylene dimethyl ether, tert-butanol, 3-methyl-1-butanol, benzyl alcohol, ethyl lactate, diethyl oxalate, diethyl malonate, dimethyl carbonate, cyclohexanone, ethylene glycol, noctanol, cyclopentanol, n-heptane, n-hexane, cyclohexane, iso-propyl ether, n-butyl ether, 1,2-propanediol, 1,3-propanediol, tetraethylene glycol, ethyl cyanoacetate and water, were chosen. These solvents were classified into "good" solvents and "poor" solvents based on the solubility of DFL in the single solvent listed above.

Evaporation. $20 \mathrm{mg}$ of Dufulin Form I were dissolved in selected "good" solvents, respectively. The clear solutions were allowed to slowly evaporate at room temperature to collect yield solid powders.

Slurry. $20 \mathrm{mg}$ of DFL Form I were added to $2 \mathrm{~mL}$ of selected "poor" solvents, 
respectively. The suspensions were placed in a mechanical shaker at a speed of $180 \mathrm{rpm}$ for $24 \mathrm{~h}$. The solid powders were obtained after filtration.

Anti-solvent. $20 \mathrm{mg}$ of DFL Form I were dissolved in selected "good" solvents, respectively. 5-10 times volume of selected "poor" solvent was added into the clear solutions till solid powders were precipitated. The solid powders were obtained after filtration.

Cooling. $50 \mathrm{mg}$ DFL Form I were dissolved in selected "good" solvents as few as possible at a higher temperature (below the boiling point of the solvent), respectively. The solutions were placing in $4^{\circ} \mathrm{C}$ condition and the solid powders were collected if precipitation out happened.

The results of selected polymorphism screening experiment were listed in Table S1.

Table S1. The summary of selected solid-state screening experiment.

\begin{tabular}{ccc}
\hline Solvents & Procedures & Results \\
\hline- & Melting Form I & Amorphous \\
$N, N$-Dimethylformamide (DMF) & Eeating amorphous & Form II \\
Tetrahydrofuran (THF) & Evaporation & Form I \\
Pyridine & Evaporation & Form I \\
Dichloromethane $(\mathrm{DCM})$ & Evaporation & Form I \\
Acetone & Evaporation & Form I \\
Ethyl acetate $(\mathrm{EA})$ & Evaporation & Form I \\
\hline
\end{tabular}




\begin{tabular}{|c|c|c|}
\hline Methanol & Evaporation & Form I \\
\hline Ethanol & Evaporation & Form I \\
\hline Water & Slurry & Form I \\
\hline n-Hexane & Slurry & Form I \\
\hline Iso-propyl ether & Slurry & Form I \\
\hline Ethylene glycol (EG) & Slurry & Form I \\
\hline Ethanol & Slurry & Form I \\
\hline 1,4-Dioxane & Slurry & Form V \\
\hline Methanol & Slurry & Form I \\
\hline DMF & Cooling & Form I \\
\hline DCM & Cooling & Form I \\
\hline Acetone & Cooling & Form I \\
\hline EA & Cooling & Form I \\
\hline n-Hexane & Cooling & Form I \\
\hline Iso-propyl ether & Cooling & Form I \\
\hline Ethanol & Cooling & Form I \\
\hline Methanol & Cooling & Form I \\
\hline 1,4-Dioxane & Cooling & Form V \\
\hline DMF/ water & Anti-solvent & Form I \\
\hline $\mathrm{DMF} / \mathrm{n}$-Hexane & Anti-solvent & Form I \\
\hline DMF/ Iso-propyl ether & Anti-solvent & Form I \\
\hline $\mathrm{DMF} / \mathrm{EG}$ & Anti-solvent & Form I \\
\hline
\end{tabular}


DMF/ Ethanol

$\mathrm{EA} /$ water

EA/ n-Hexane

EA/ Iso-propyl ether

EA/ EG

EA/ Ethanol

$\mathrm{THF} /$ water

THF/ n-Hexane

THF/ Iso-propyl ether

THF/ EG

THF/ Ethanol

$\mathrm{DCM} /$ water

DCM/ n-Hexane

$\mathrm{DCM} /$ Iso-propyl ether

DCM/ EG

DCM/ Ethanol

Acetone/ water

Acetone/ n-Hexane

Acetone/ Iso-propyl ether

Acetone/ EG

Acetone/ Ethanol
Anti-solvent

Form I

Anti-solvent

Form I

Anti-solvent

Form I

Anti-solvent

Form I

Anti-solvent

Form I

Anti-solvent

Form I

Anti-solvent

Form I

Anti-solvent

Form III

Anti-solvent

Form I

Anti-solvent

Form I

Anti-solvent

Form I

Anti-solvent

Anti-solvent

Form VI

Form I

Anti-solvent

Form I

Anti-solvent

Form I

Anti-solvent

Anti-solvent

Form I

Anti-solvent

Form I

Anti-solvent

Form I

Anti-solvent

Form IV

Anti-solvent

Form I 
Table S2. The detailed data of DFL polymorphs peaks.

\begin{tabular}{|c|c|c|c|c|c|c|c|c|c|c|c|}
\hline \multicolumn{2}{|c|}{ Form I } & \multicolumn{2}{|c|}{ Form II } & \multicolumn{2}{|c|}{ Form III } & \multicolumn{2}{|c|}{ Form IV } & \multicolumn{2}{|c|}{ Form V } & \multicolumn{2}{|c|}{ Form VI } \\
\hline $2 \theta$ & $I($ Height $) \%$ & $2 \theta$ & $I($ Height $) \%$ & $2 \theta$ & $I($ Height $) \%$ & $2 \theta$ & $I($ Height $) \%$ & $2 \theta$ & $I($ Height $) \%$ & $2 \theta$ & $I($ Height $) \%$ \\
\hline 8.1 & 24.5 & 8.7 & 20.0 & 6.6 & 3.6 & 7.6 & 16.0 & 9.3 & 90.2 & 8.1 & 2.5 \\
\hline 10.4 & 7.0 & 9.3 & 15.1 & 9.4 & 14.6 & 8.6 & 4.0 & 10.3 & 72.9 & 8.6 & 29.7 \\
\hline 11.8 & 8.4 & 9.8 & 4.2 & 10.1 & 10.2 & 9.3 & 79.3 & 10.5 & 23.9 & 9.2 & 3.6 \\
\hline 12.3 & 2.0 & 11.9 & 91.5 & 11.6 & 5.9 & 11.4 & 73.7 & 13.0 & 27.3 & 10.3 & 14.5 \\
\hline 12.7 & 24.3 & 12.5 & 10.6 & 12.2 & 60.4 & 13.2 & 2.2 & 13.3 & 40.9 & 10.7 & 5.1 \\
\hline 13.1 & 2.2 & 12.9 & 13.7 & 12.8 & 37.4 & 14.7 & 49.1 & 15.9 & 31.9 & 11.8 & 26.2 \\
\hline 15.4 & 24.1 & 13.3 & 2.5 & 13.1 & 16.0 & 15.2 & 22.9 & 16.1 & 14.9 & 12.2 & 14.7 \\
\hline 16.0 & 100.0 & 14.9 & 100.0 & 14.2 & 20.9 & 16.1 & 26.8 & 16.4 & 5.0 & 13.0 & 14.8 \\
\hline 16.4 & 5.7 & 16.2 & 29.8 & 14.5 & 5.2 & 16.6 & 16.4 & 17.1 & 2.3 & 13.6 & 2.8 \\
\hline 17.4 & 7.5 & 17.3 & 56.7 & 15.5 & 11.2 & 16.8 & 48.1 & 17.6 & 40.0 & 14.6 & 19.1 \\
\hline 18.1 & 12.0 & 18.0 & 8.8 & 16.3 & 39.2 & 17.2 & 2.5 & 17.8 & 36.4 & 15.1 & 100.0 \\
\hline 19.2 & 8.6 & 18.4 & 9.8 & 17.7 & 29.4 & 18.1 & 18.1 & 19.0 & 30.8 & 16.2 & 29.6 \\
\hline 20.0 & 8.0 & 18.9 & 17.4 & 18.4 & 19.6 & 18.5 & 11.3 & 20.8 & 34.1 & 16.7 & 12.4 \\
\hline 20.6 & 16.9 & 19.8 & 23.0 & 18.9 & 36.9 & 19.9 & 56.8 & 21.3 & 10.2 & 17.4 & 27.3 \\
\hline 20.8 & 8.2 & 20.3 & 46.3 & 19.5 & 14.7 & 20.8 & 24.7 & 22.2 & 15.5 & 17.6 & 26.9 \\
\hline 21.3 & 3.2 & 21.4 & 22.9 & 19.8 & 15.0 & 21.3 & 81.5 & 23.6 & 26.6 & 18.5 & 7.4 \\
\hline 22.2 & 9.8 & 22.0 & 5.1 & 20.3 & 39.9 & 21.5 & 16.0 & 24.2 & 4.7 & 19.2 & 7.8 \\
\hline 22.8 & 4.3 & 22.4 & 84.6 & 21.6 & 68.9 & 22.3 & 100.0 & 25.8 & 44.1 & 19.6 & 2.5 \\
\hline 23.1 & 32.9 & 22.7 & 46.4 & 22.2 & 16.8 & 22.9 & 27.2 & 26.9 & 22.3 & 20.2 & 17.6 \\
\hline 23.4 & 7.0 & 24.1 & 9.9 & 23.5 & 53.3 & 23.6 & 9.8 & 27.1 & 24.1 & 20.6 & 12.6 \\
\hline 23.6 & 27.0 & 24.8 & 3.6 & 24.0 & 100.0 & 24.0 & 27.2 & 27.3 & 24.2 & 21.0 & 22.6 \\
\hline- & - & 25.2 & 3.7 & 24.6 & 4.3 & 24.4 & 13.9 & 28.3 & 3.9 & 21.6 & 2.4 \\
\hline- & - & 26.4 & 14.2 & 25.1 & 5.5 & 24.7 & 13.4 & 29.1 & 7.8 & 22.7 & 85.0 \\
\hline- & - & 27.1 & 12.1 & 25.9 & 15.0 & 25.2 & 82.4 & 29.7 & 2.7 & 23.4 & 2.9 \\
\hline- & - & - & - & 26.1 & 25.3 & 26.6 & 10.8 & 31.0 & 14.0 & 24.2 & 2.2 \\
\hline- & - & - & - & 26.6 & 7.1 & 27.2 & 3.6 & 32.1 & 100.0 & 24.7 & 4.9 \\
\hline
\end{tabular}




\begin{tabular}{|c|c|c|c|c|c|c|c|c|c|c|c|}
\hline- & - & - & - & 27.1 & 6.7 & 27.6 & 11.7 & 34.8 & 6.7 & 26.2 & 23.7 \\
\hline- & - & - & - & 27.7 & 2.2 & 28.1 & 18.6 & 36.2 & 2.8 & 27.3 & 7.8 \\
\hline- & - & - & - & 28.5 & 5.0 & 28.4 & 8.0 & 36.5 & 6.8 & 27.8 & 3.5 \\
\hline- & - & - & - & 28.9 & 6.7 & 29.0 & 2.9 & 36.9 & 6.2 & 28.0 & 8.5 \\
\hline- & - & - & - & 29.7 & 3.2 & 29.7 & 3.8 & 37.5 & 2.0 & 28.9 & 3.1 \\
\hline- & - & - & - & 30.1 & 1.8 & 30.3 & 17.1 & 38.3 & 2.6 & 29.6 & 3.2 \\
\hline- & - & - & - & 30.9 & 40.2 & 30.7 & 8.4 & 39.0 & 6.1 & 31.0 & 4.7 \\
\hline- & - & - & - & 31.3 & 3.4 & 31.0 & 3.7 & 42.2 & 5.9 & 32.9 & 2.7 \\
\hline- & - & - & - & 31.7 & 6.6 & 32.5 & 6.5 & 42.7 & 2.3 & 33.4 & 1.7 \\
\hline- & - & - & - & 32.9 & 1.8 & 32.9 & 3.4 & 43.1 & 12.7 & 34.3 & 4.9 \\
\hline- & - & - & - & 34.4 & 9.7 & 33.4 & 6.0 & 44.3 & 4.3 & 34.9 & 1.8 \\
\hline- & - & - & - & 34.7 & 5.1 & 34.2 & 2.6 & - & - & 35.6 & 1.8 \\
\hline- & - & - & - & 35.7 & 13.2 & 34.6 & 6.1 & - & - & 36.7 & 2.5 \\
\hline- & - & - & - & 36.3 & 4.6 & 35.0 & 4.9 & - & - & 37.2 & 3.1 \\
\hline- & - & - & - & 36.5 & 4.1 & 35.8 & 13.7 & - & - & 39.1 & 1.8 \\
\hline- & - & - & - & 37.3 & 3.5 & 36.2 & 5.3 & - & - & 39.5 & 2.0 \\
\hline- & - & - & - & 37.9 & 5.0 & 36.5 & 3.7 & - & - & 40.0 & 2.0 \\
\hline- & - & - & - & 39.0 & 5.4 & 37.4 & 1.9 & - & - & 41.2 & 2.5 \\
\hline- & - & - & - & 40.6 & 2.5 & 38.0 & 2.7 & - & - & 41.8 & 1.3 \\
\hline- & - & - & - & 41.3 & 4.0 & 38.6 & 1.8 & - & - & 43.0 & 2.7 \\
\hline- & - & - & - & 41.6 & 6.7 & 39.3 & 2.1 & - & - & 44.6 & 1.2 \\
\hline- & - & - & - & 42.5 & 1.4 & 39.7 & 2.2 & - & - & - & - \\
\hline- & - & - & - & 42.8 & 2.2 & 40.1 & 3.0 & - & - & - & - \\
\hline- & - & - & - & 43.9 & 6.0 & 40.6 & 2.2 & - & - & - & - \\
\hline- & - & - & - & 44.5 & 2.0 & 41.1 & 3.9 & - & - & - & - \\
\hline- & - & - & - & - & - & 42.1 & 3.7 & - & - & - & - \\
\hline- & - & - & - & - & - & 43.5 & 3.4 & - & - & - & - \\
\hline- & - & - & - & - & - & 44.5 & 1.5 & - & - & - & - \\
\hline
\end{tabular}




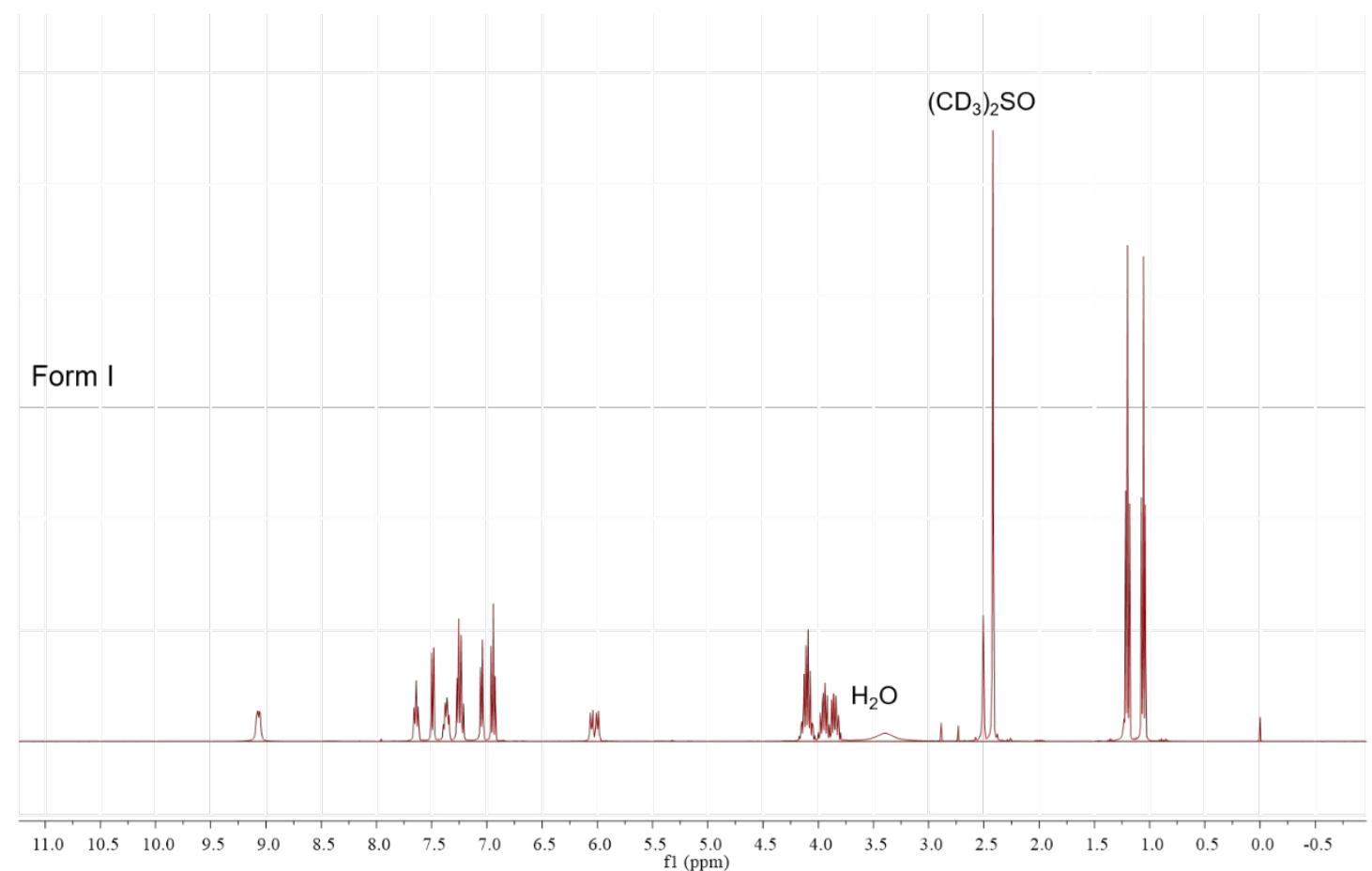

(a)

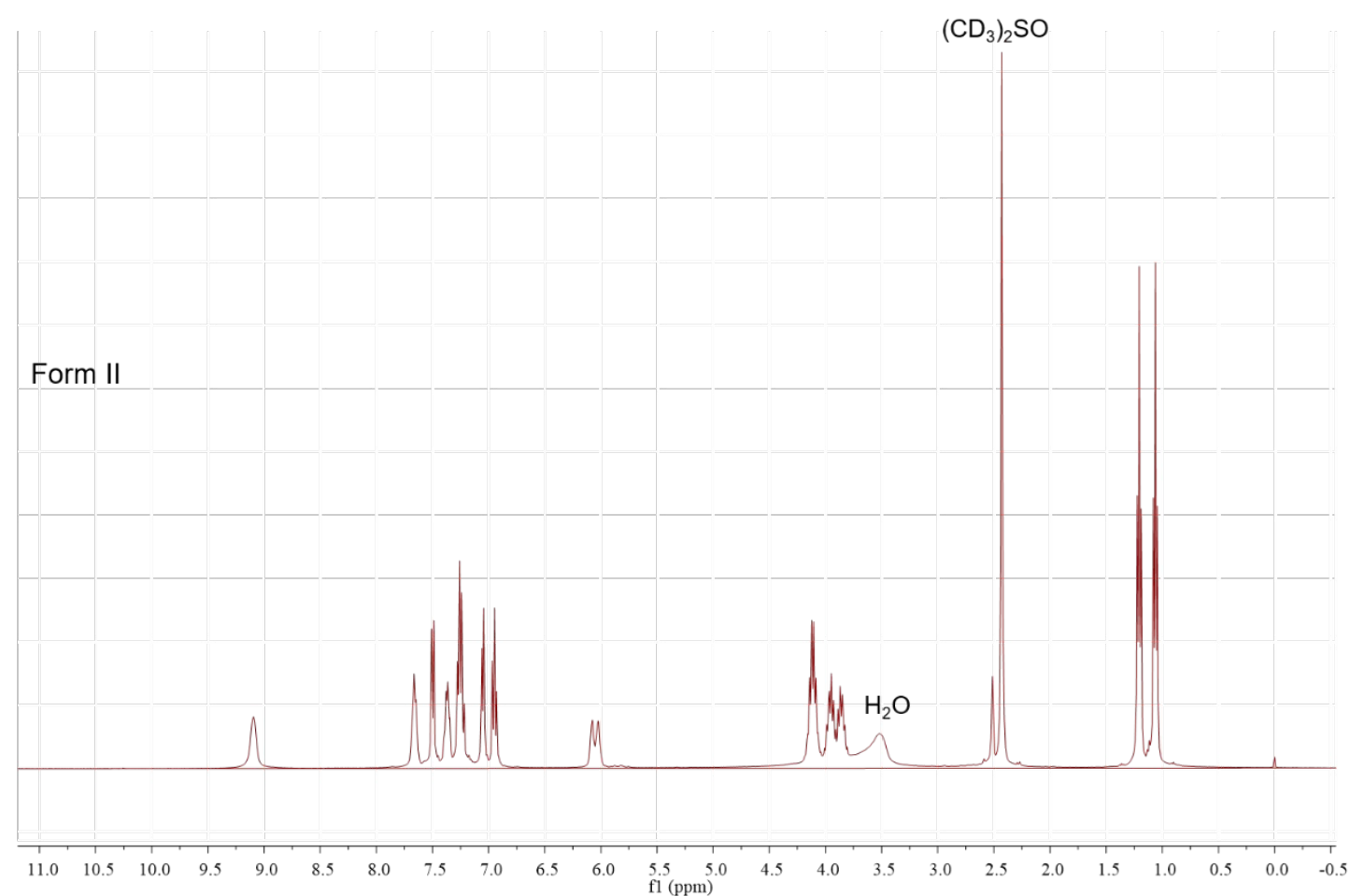

(b) 


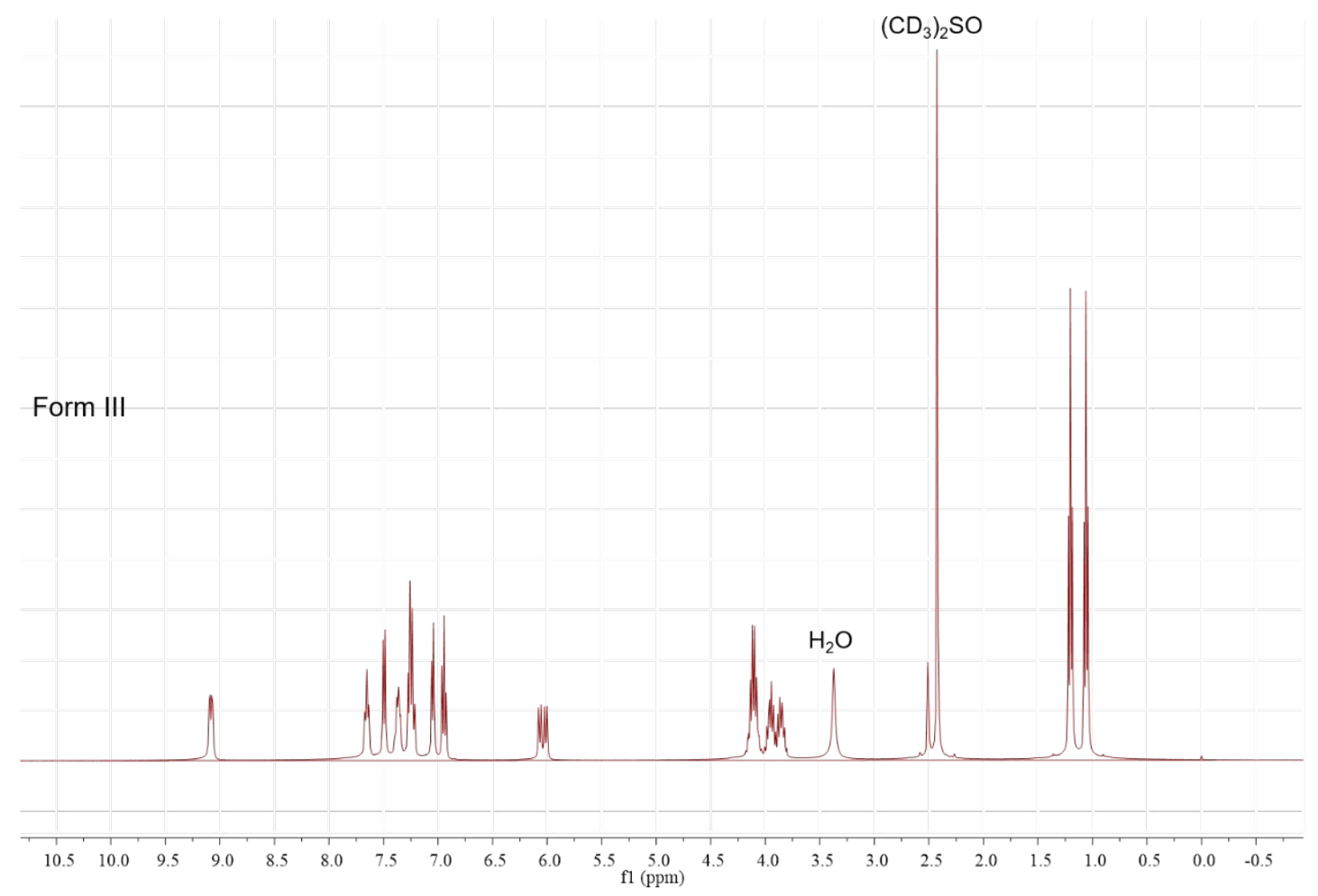

(c)

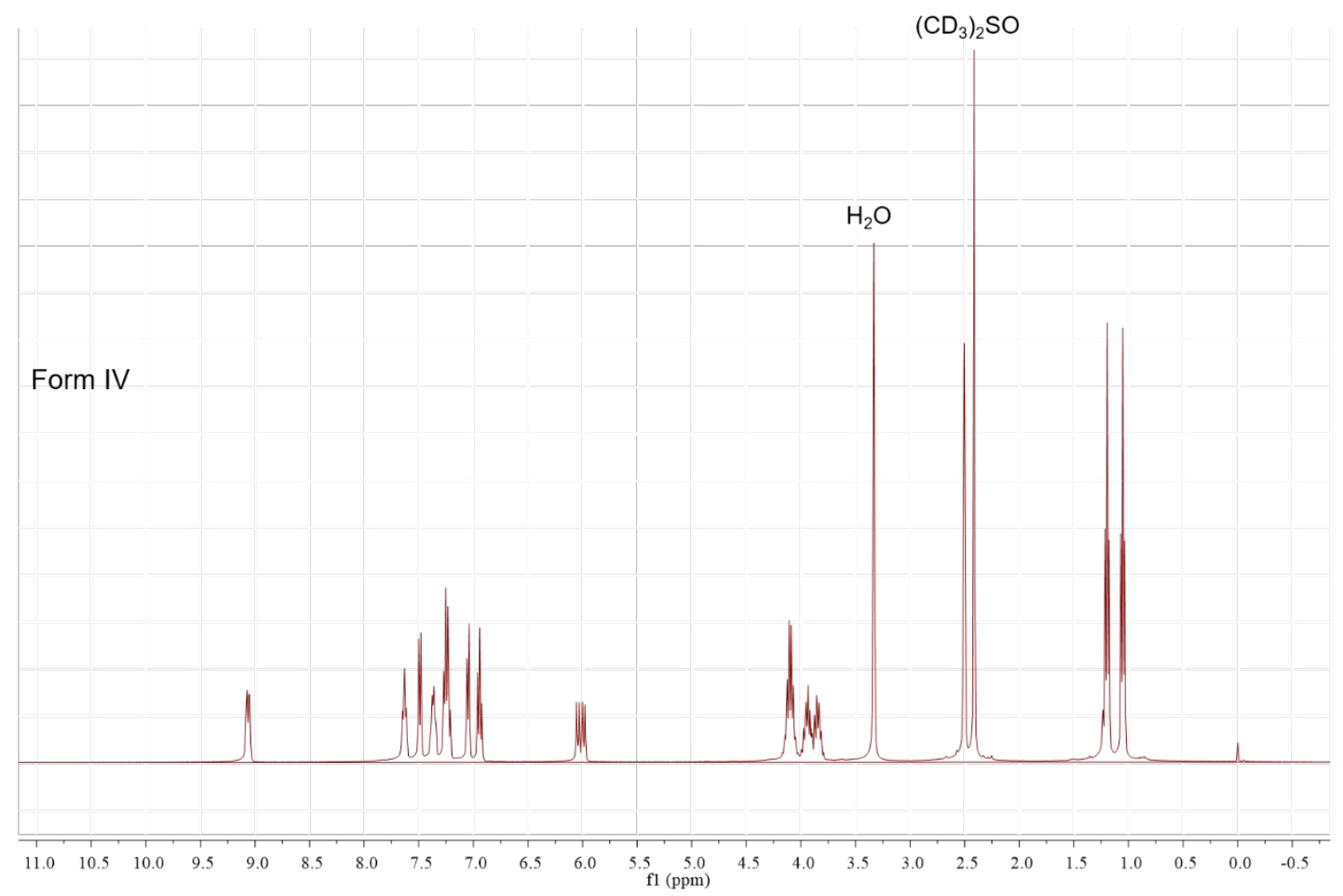

(d) 


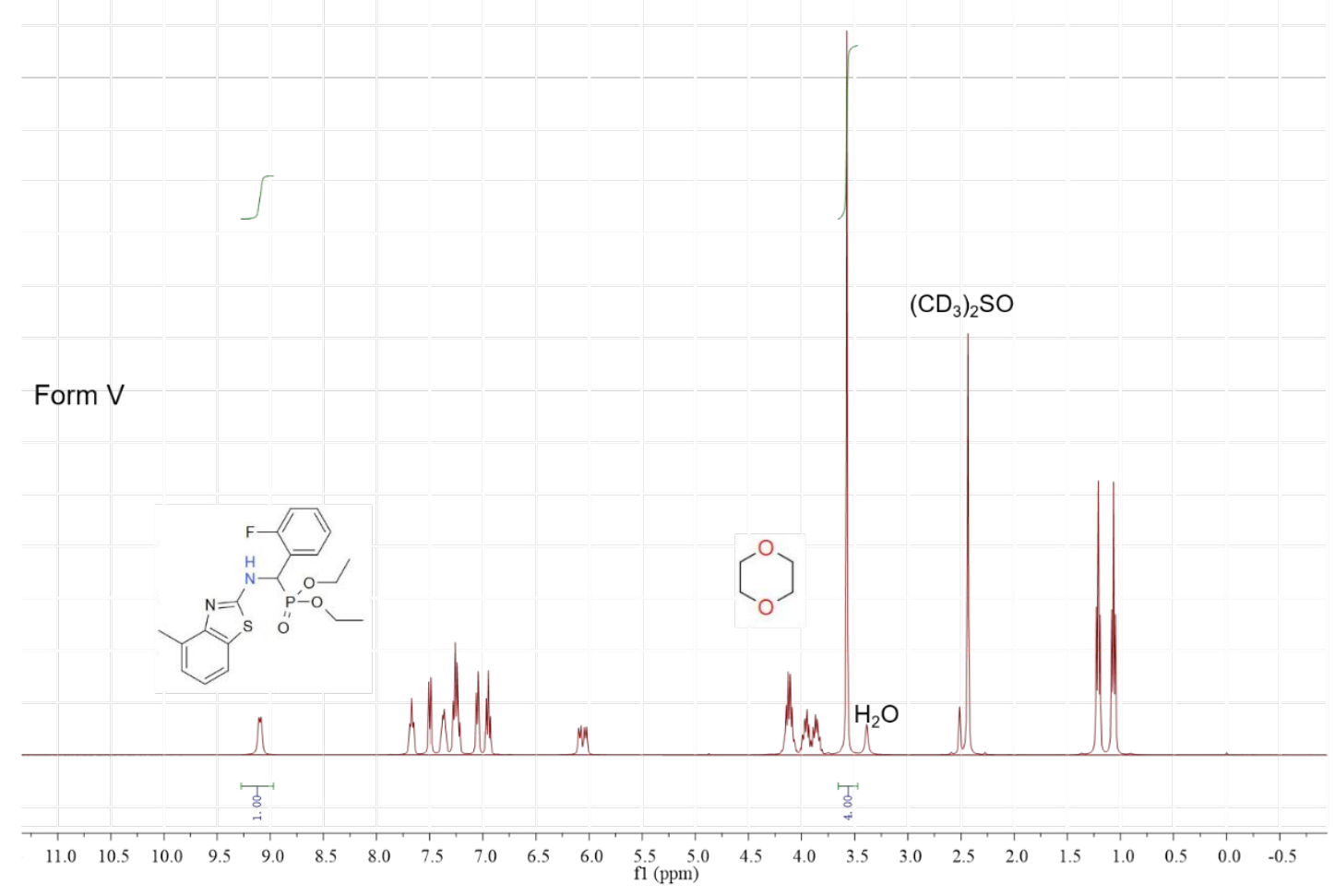

(e)

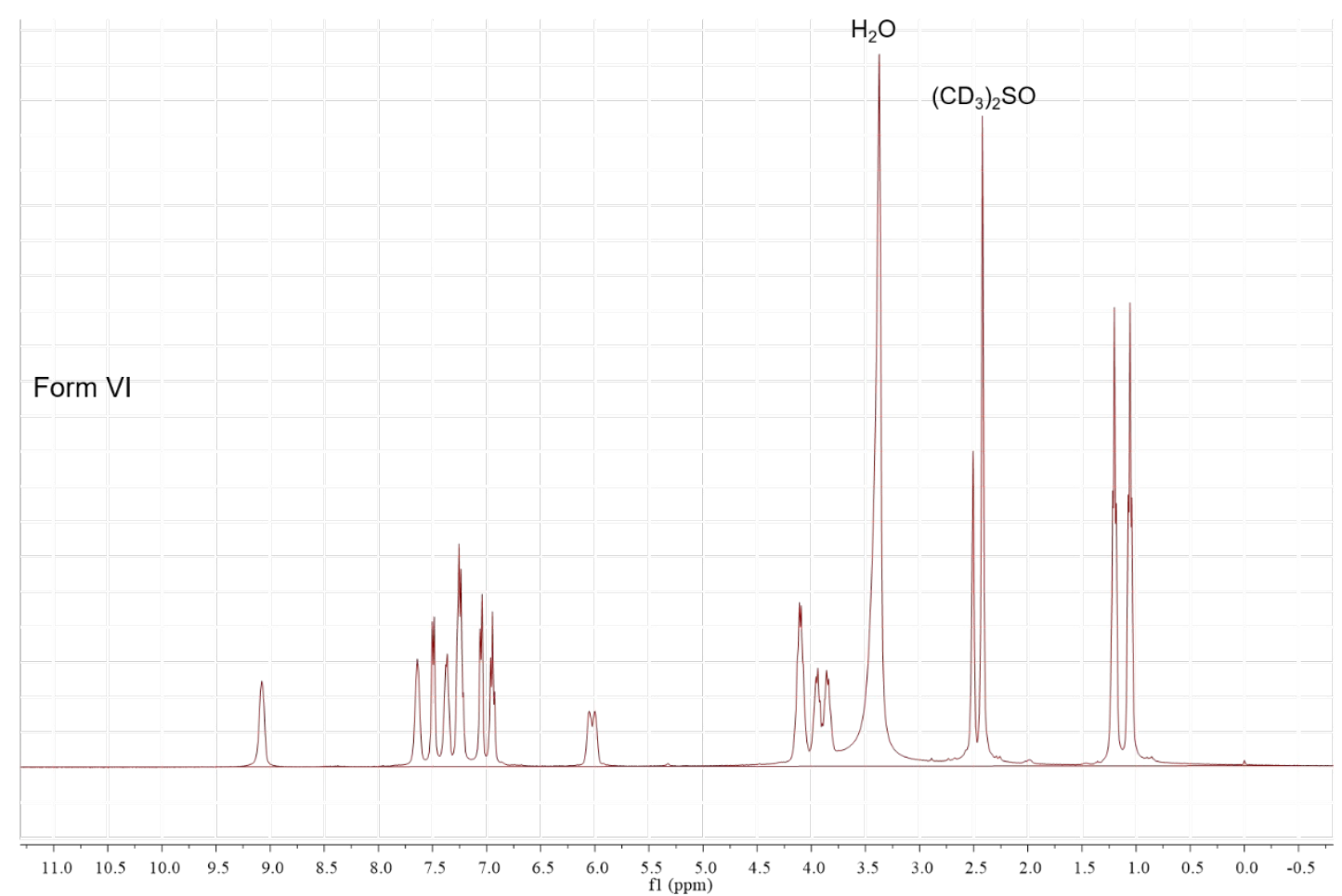

(f) 


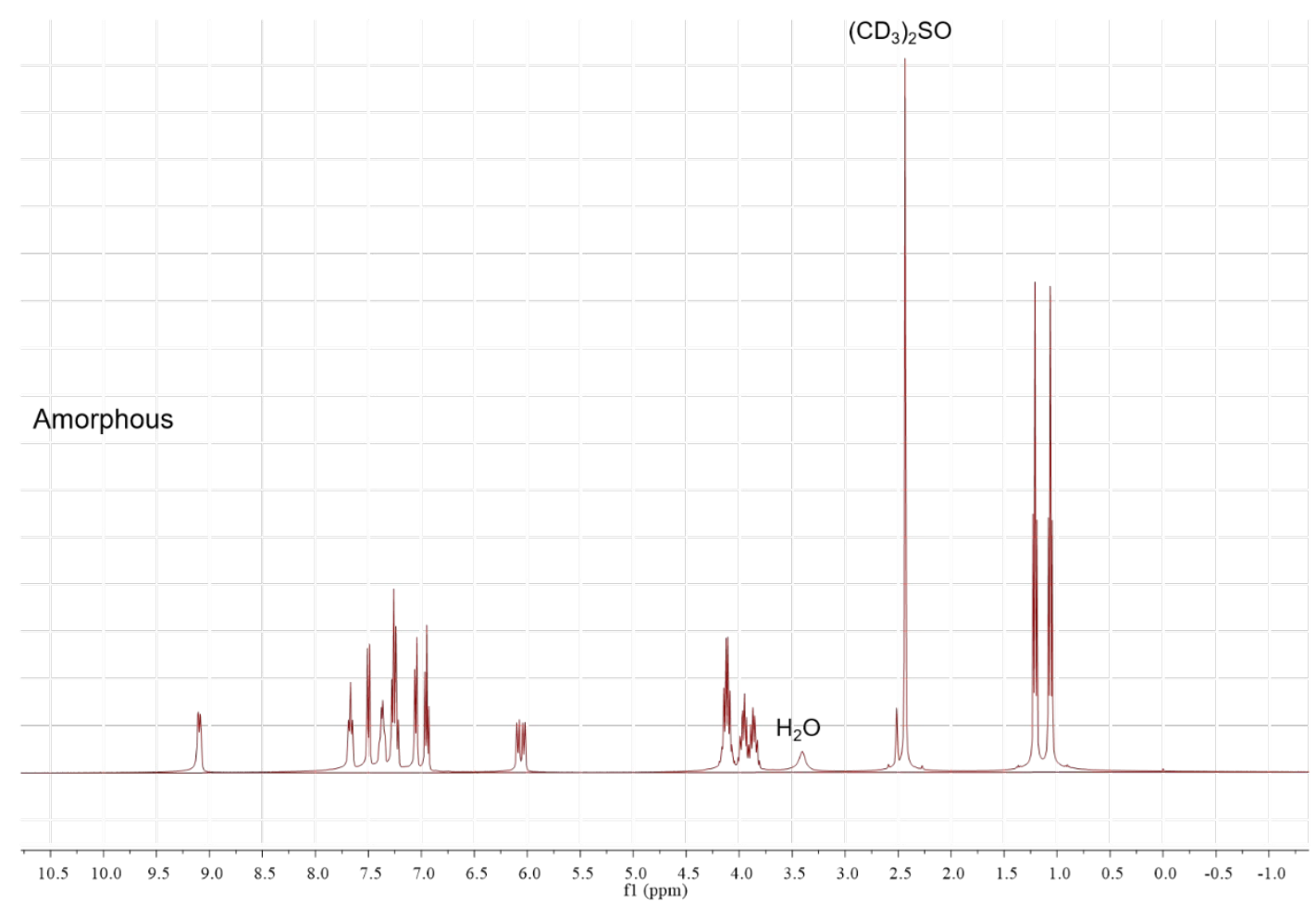

(g)

Figure S2. ${ }^{1} \mathrm{H}$ NMR spectra of DFL polymorphs. (a) Form I, (b) Form II, (c) Form III, (d) Form IV, (e) Form V, (f) Form VI, and (g) amorphous.

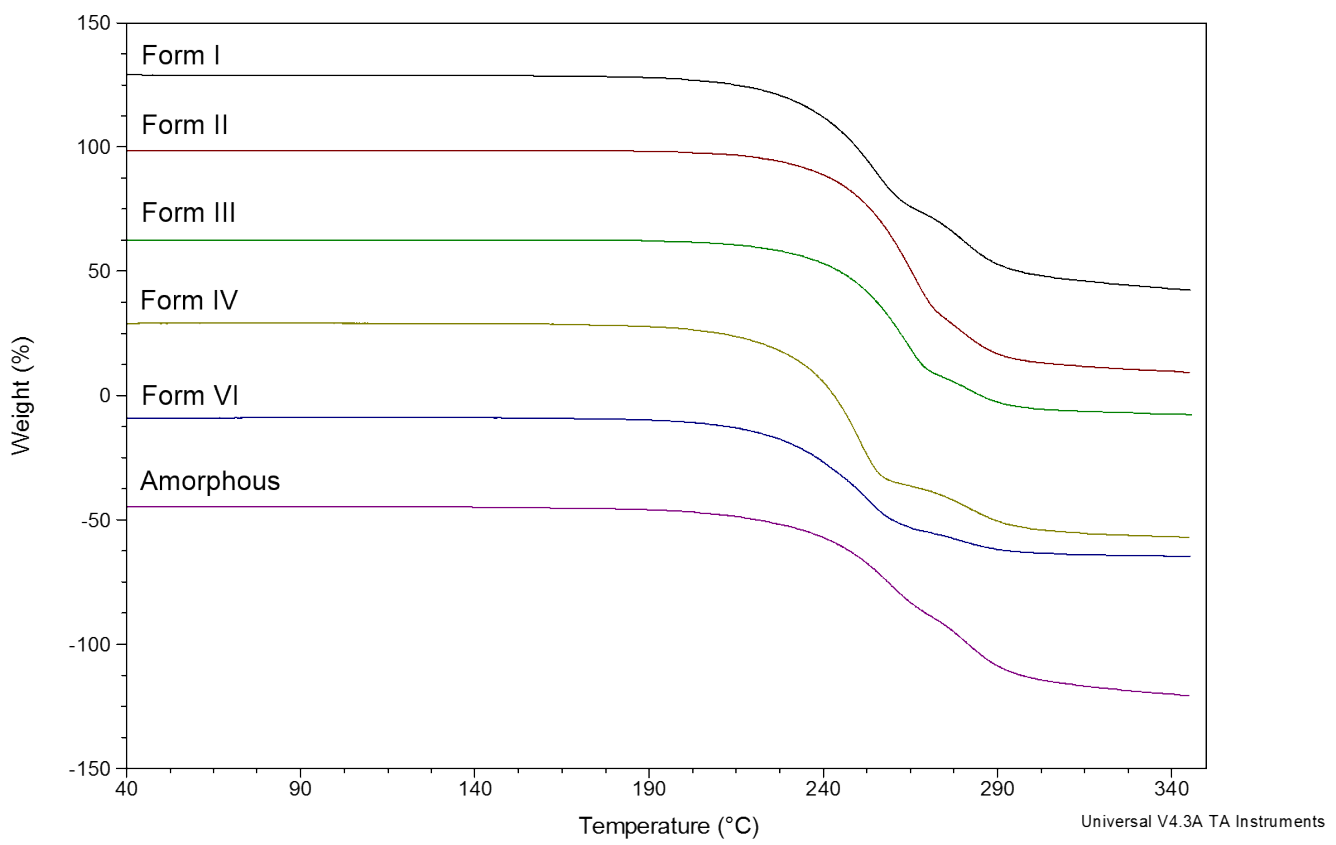


Figure S3. TGA curves for Form I-IV, VI and amorphous of DFL.

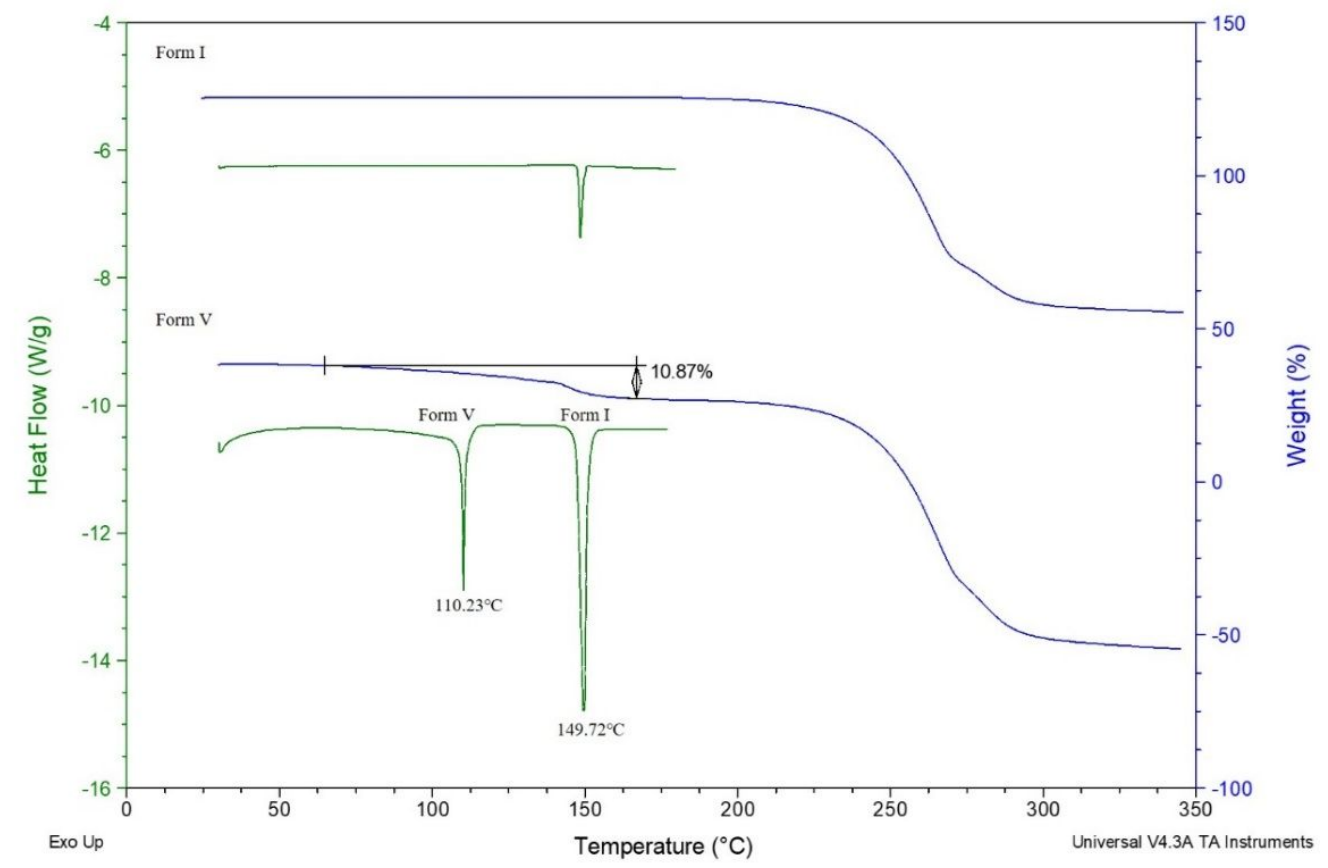

(a)

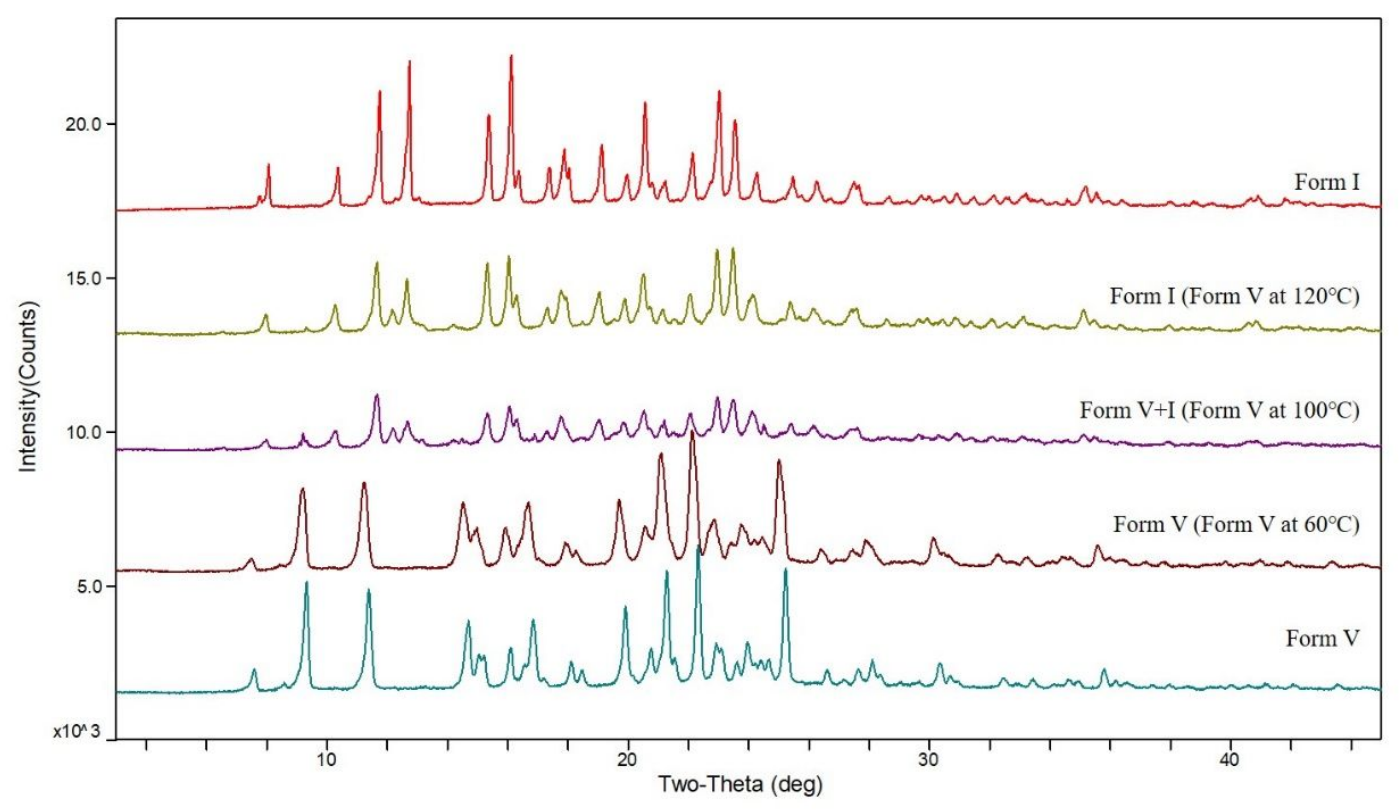

(b)

Figure S4. Representative DSC and TGA curve (a) and the PXRD of desolvation 
process (b) for DFL Form V.

(a)

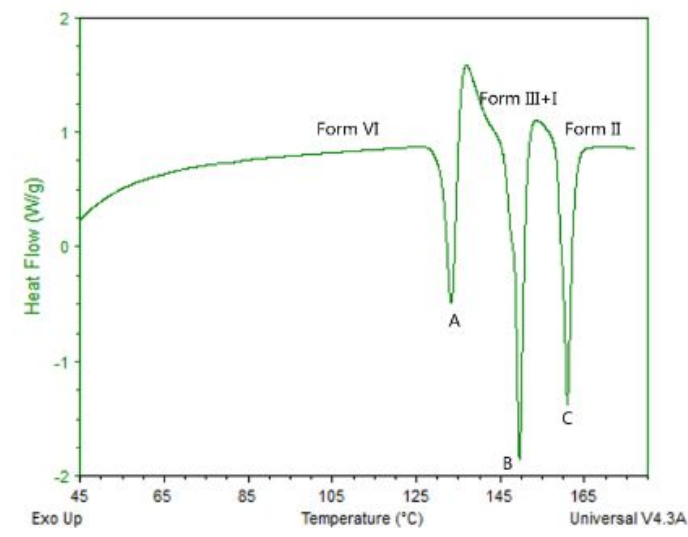

(b)

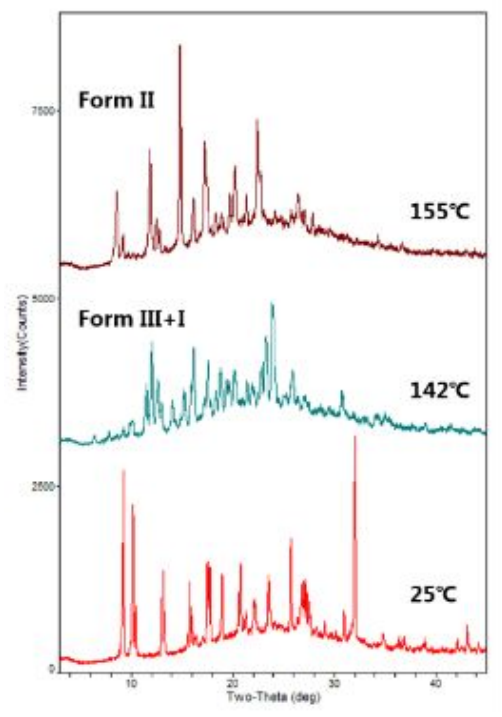

Figure S5. (a) Representative DSC curve for DFL Form VI, (b) PXRD showing the sequence of events starting with DFL Form VI.

(a)

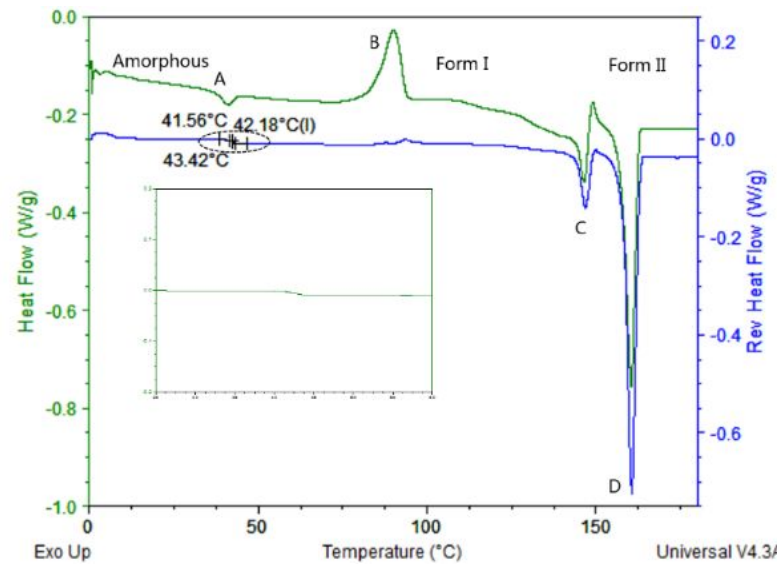

(b)

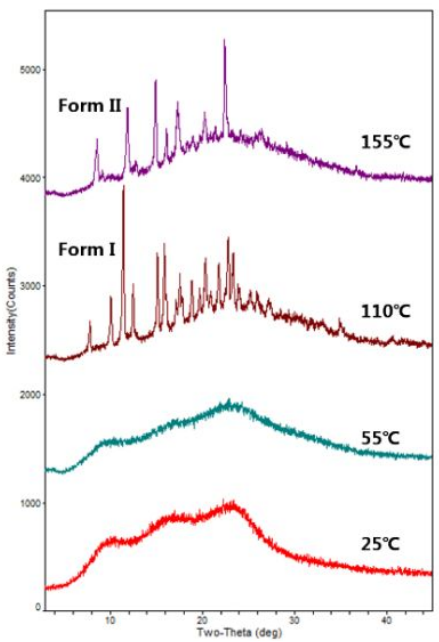

Figure S6. (a) Representative DSC curve (green) and MDSC (blue) for DFL Amorphous, (b) PXRD showing the sequence of events starting with DFL amorphous. 


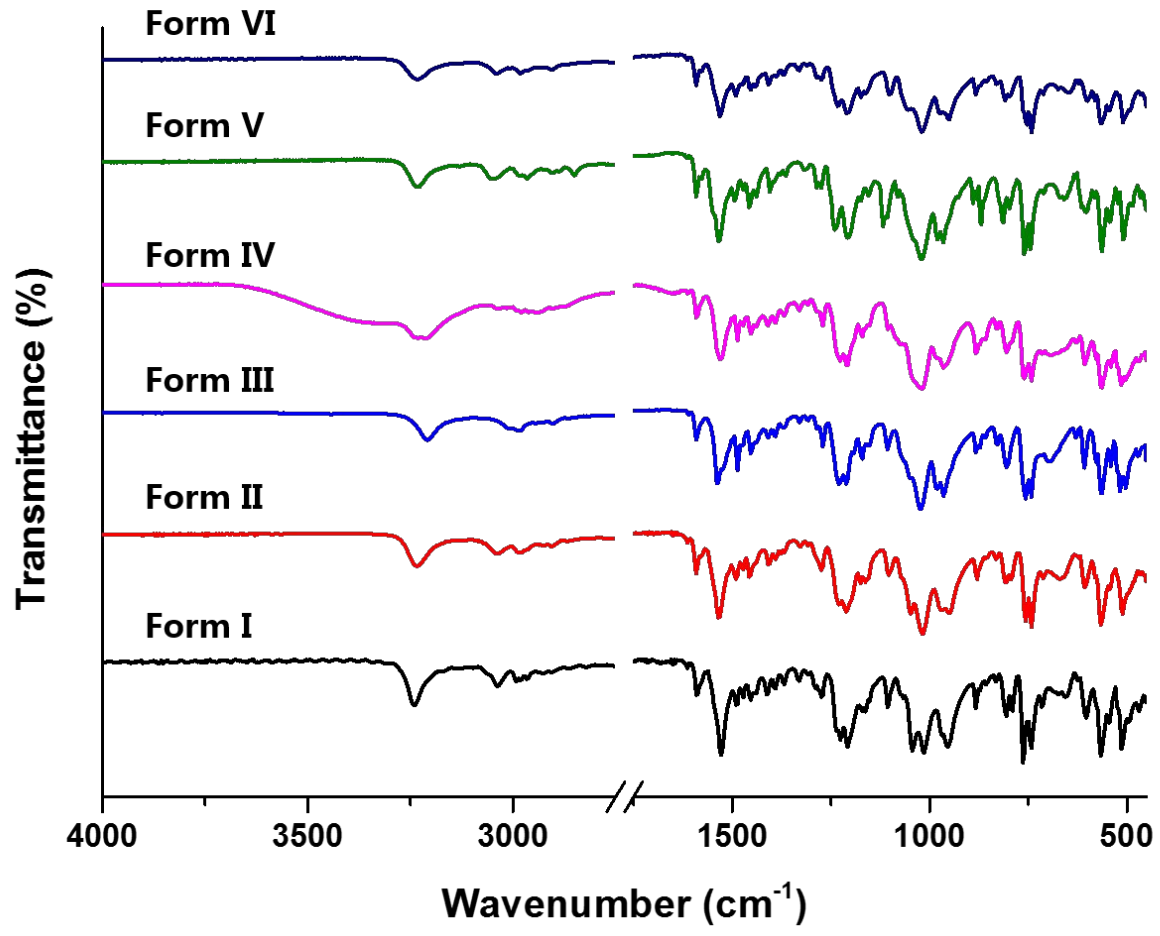

Figure S7. FT-IR spectra of the various forms of DFL.

Table S3. FT-IR spectra characteristic peaks of the various forms of DFL.

\begin{tabular}{ccccccc}
\hline \multicolumn{7}{c}{ Shift $\left(\mathrm{cm}^{-1}\right)$} \\
\cline { 1 - 4 } Form I & Form II & Form III & Form IV & Form V & Form VI & \\
\hline 3240 & 3234 & 3208 & 3222 & 3234 & 3233 & N-H stretch \\
1589,1528 & 1585,1534 & 1591,1537 & 1590,1530 & 1588,1534 & 1591,1531 & C=N stretch \\
1208,1043 & 1211,1018 & 1229,1023 & 1226,1020 & $1207,1117,1021$ & 1209,1020 & C-O stretch \\
\hline
\end{tabular}




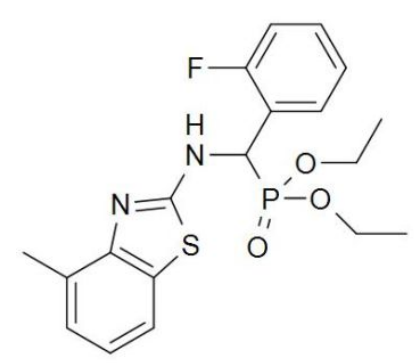

Dufulin

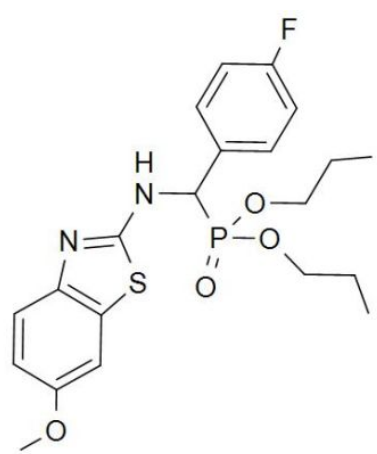

CCDC 243913

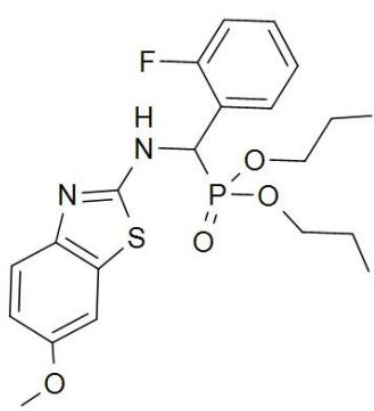

$\operatorname{CCDC} 234280$

Figure S8. The molecular structures of DFL and structurally similar molecules.

Table S4. The crystal structure data of structurally similar molecules.

\begin{tabular}{|c|c|c|}
\hline & CCDC 243913 & CCDC 234280 \\
\hline Formula & $\mathrm{C}_{21} \mathrm{H}_{26} \mathrm{FN}_{2} \mathrm{O}_{4} \mathrm{PS}$ & $\mathrm{C}_{21} \mathrm{H}_{26} \mathrm{FN}_{2} \mathrm{O}_{4} \mathrm{PS}$ \\
\hline Weight $\left(\mathrm{g} / \mathrm{mol}^{-1}\right)$ & 452.47 & 452.47 \\
\hline Crystal system & Tetragonal & Triclinic \\
\hline Space group & $\mathrm{I} 4(1) / \mathrm{a}$ & P-1 \\
\hline$a(\AA)$ & $21.352(3)$ & $9.601(3)$ \\
\hline$b(\AA)$ & $21.352(3)$ & $10.547(4)$ \\
\hline$c(\AA)$ & $20.162(5)$ & $11.894(4)$ \\
\hline$\alpha(\operatorname{deg})$ & 90.00 & $97.902(6)$ \\
\hline$\beta(\operatorname{deg})$ & 90.00 & $105.520(5)$ \\
\hline$\gamma(\operatorname{deg})$ & 90.00 & $91.851(5)$ \\
\hline Volume $\left(\AA^{3}\right)$ & $9192(3)$ & $1146.5(7)$ \\
\hline Z & 16 & 2 \\
\hline
\end{tabular}




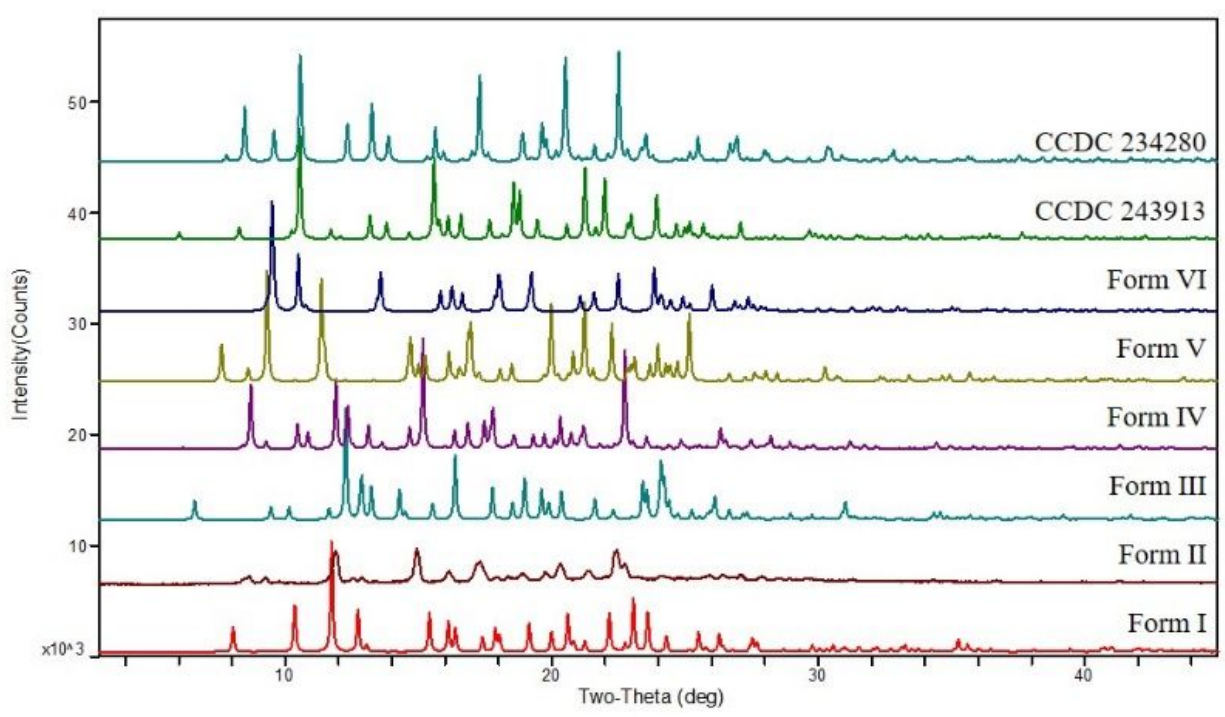

Figure S9. The simulated powder pattern of structurally similar molecules.

CCDC 234280
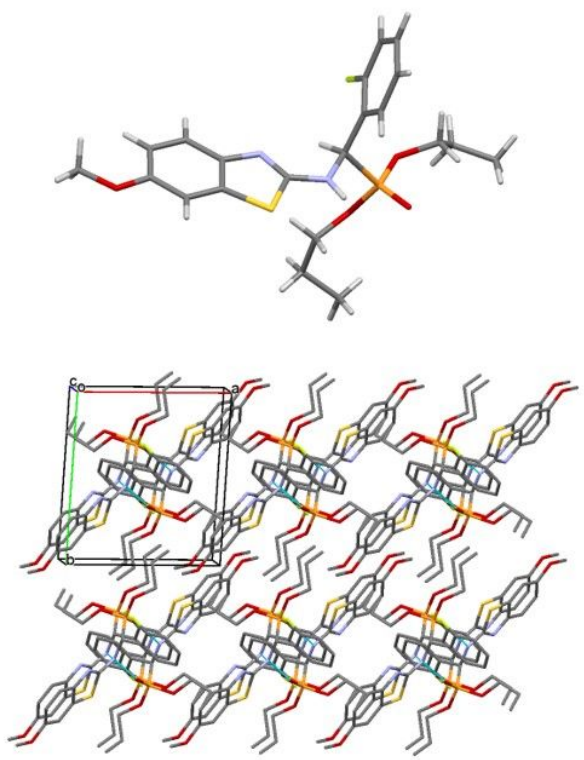

CCDC 243913
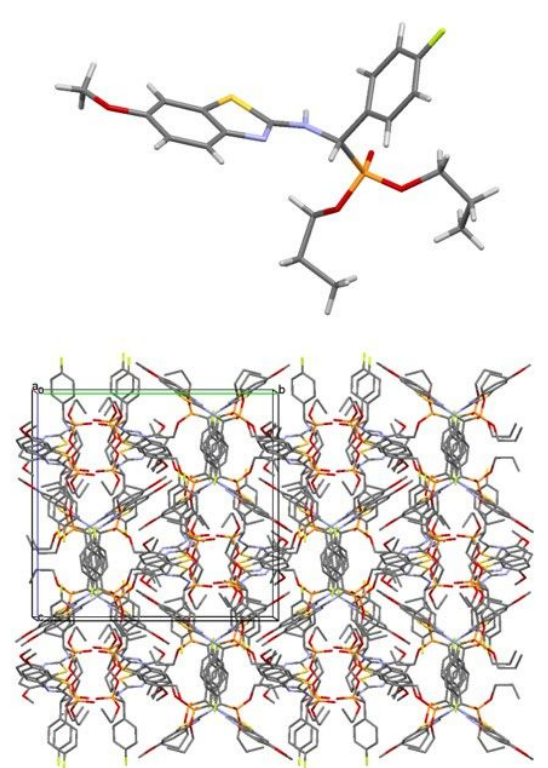

Figure S10. The crystal structure of structurally similar molecules. 


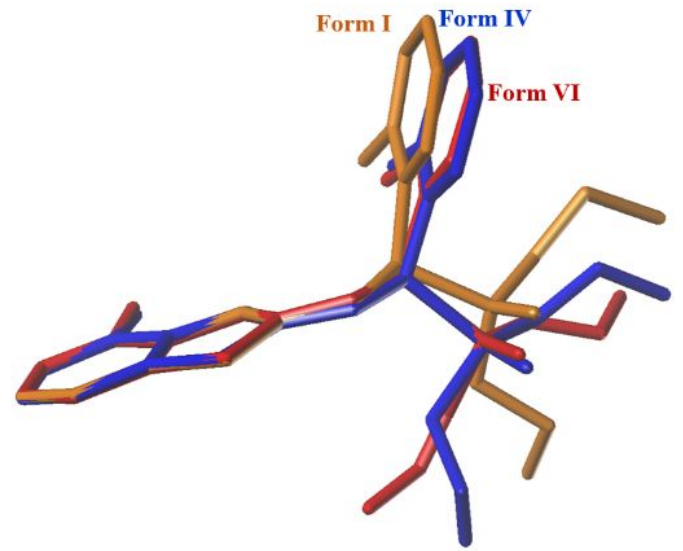

(a)

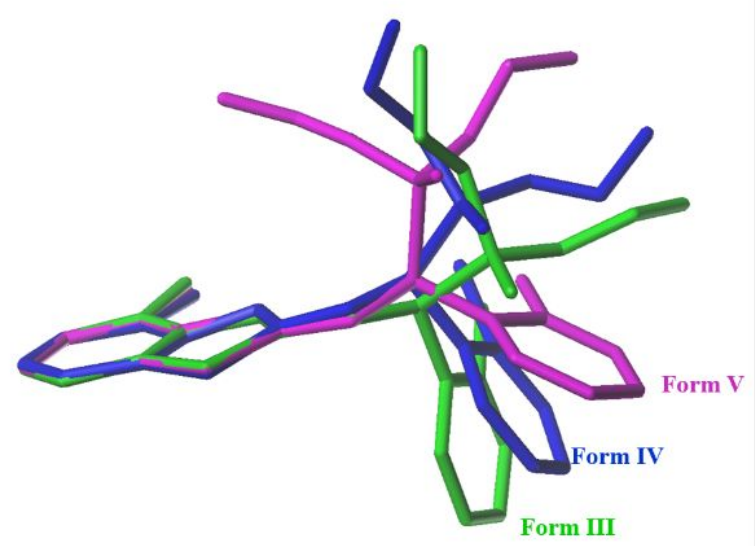

(b)

Figure S11. Overlay of the DFL molecules (a) orange: Form I (R), blue: Form IV (R), red: Form VI (R), (b) green: Form III (S), blue: Form IV (S), and magenta: Form V (S).

Table S5. The detailed data of torsion angles.

\begin{tabular}{ccccccc}
\hline Torsion angle $\left(^{\circ}\right)$ & Form I & Form III & Form IV-R & Form IV-S & Form V & Form VI \\
\hline O1-P1-C9-N2 & $51.4(5)$ & $49.8(2)$ & $50.5(8)$ & $47(1)$ & $-60.0(3)$ & $46.6(6)$ \\
O1-P1-C9-C10 & $-75.2(5)$ & $-76.4(2)$ & $-79(1)$ & $-76(1)$ & $64.9(3)$ & $-80.9(6)$ \\
O2-P1-C9-N2 & $-75.3(5)$ & $177.3(4)$ & $-171.2(6)$ & $-84.8(4)$ & $164.7(6)$ & $-77.5(4)$ \\
O2-P1-C9-C10 & $158.2(5)$ & $51.1(5)$ & $59.5(9)$ & $151.8(7)$ & $-70.4(6)$ & $155.0(4)$ \\
O3-P1-C9-N2 & $176.5(4)$ & $-75.9(4)$ & $-60.3(3)$ & $176.3(7)$ & $77.5(5)$ & $-172(1)$ \\
O3-P1-C9-C10 & $50.0(5)$ & $157.9(4)$ & $170.4(8)$ & $53(1)$ & $-157.6(5)$ & $61(1)$ \\
C7-N1-C8-S1 & $0.6(6)$ & $0.9(3)$ & $0.6(9)$ & $-5(4)$ & $-0.6(5)$ & $-1.2(6)$ \\
C7-N1-C8-N2 & $-176.2(5)$ & $-177.2(3)$ & $-178.2(9)$ & $-179(3)$ & $179.1(4)$ & $-178.4(5)$ \\
H2-N2-C9-P1 & -40.6 & -21.8 & -55.7 & -37.7 & 79.9 & -57.1 \\
H2-N2-C9-C10 & 82.7 & 104.3 & 71.8 & 86.9 & -43.5 & 67.6 \\
C8-N2-C9-P1 & $139.3(5)$ & $158.2(2)$ & $124.5(3)$ & $142(2)$ & $-100.2(4)$ & $122.9(5)$ \\
C8-N2-C9-C10 & $-97.4(7)$ & $-75.6(3)$ & $-108.0(8)$ & $-93(2)$ & $136.4(4)$ & $-112.4(6)$ \\
C1-C2-C3-H3 & 3 & 3 & 4 & -1 & 2.6 & -1 \\
C1-C2-C3-C4 & $-177.3(9)$ & $177.2(8)$ & $-176(2)$ & $179(2)$ & $-177.4(6)$ & $178.9(8)$ \\
\hline
\end{tabular}




\begin{tabular}{|c|c|c|c|c|c|c|}
\hline $\mathrm{C} 7-\mathrm{C} 2-\mathrm{C} 3-\mathrm{H} 3$ & -178.8 & -179.4 & -180 & 180 & -178.7 & -178.3 \\
\hline $\mathrm{C} 7-\mathrm{C} 2-\mathrm{C} 3-\mathrm{C} 4$ & $1(1)$ & $0.6(6)$ & $-0(3)$ & $0(2)$ & $1.2(9)$ & $2(1)$ \\
\hline $\mathrm{C} 1-\mathrm{C} 2-\mathrm{C} 7-\mathrm{N} 1$ & $-1(1)$ & $-2.8(9)$ & $2(3)$ & $15(3)$ & $-2.3(7)$ & $2(1)$ \\
\hline $\mathrm{C} 1-\mathrm{C} 2-\mathrm{C} 7-\mathrm{C} 6$ & $178.3(7)$ & $177.0(8)$ & $176(2)$ & $-179(2)$ & $177.0(5)$ & $-176.6(6)$ \\
\hline $\mathrm{C} 3-\mathrm{C} 2-\mathrm{C} 7-\mathrm{N} 1$ & $-179.3(6)$ & $179.3(3)$ & $-174(2)$ & $-167(2)$ & $179.0(5)$ & $179.2(6)$ \\
\hline $\mathrm{C} 3-\mathrm{C} 2-\mathrm{C} 7-\mathrm{C} 6$ & $-0(1)$ & $-0.8(5)$ & $0(3)$ & $-0(3)$ & $-1.7(7)$ & $0.6(9)$ \\
\hline $\mathrm{C} 2-\mathrm{C} 3-\mathrm{C} 4-\mathrm{H} 4$ & 177.6 & 179.5 & -180 & 180 & -179.9 & 177.8 \\
\hline $\mathrm{C} 2-\mathrm{C} 3-\mathrm{C} 4-\mathrm{C} 5$ & $-2(1)$ & $-0.5(7)$ & $0(2)$ & $-0(2)$ & $0(1)$ & $-2(1)$ \\
\hline $\mathrm{H} 3-\mathrm{C} 3-\mathrm{C} 4-\mathrm{H} 4$ & -2 & -0.5 & -0 & 0 & 0 & -2 \\
\hline $\mathrm{H} 3-\mathrm{C} 3-\mathrm{C} 4-\mathrm{C} 5$ & 177.8 & 179.5 & 180 & -180 & -179.9 & 177.8 \\
\hline $\mathrm{C} 3-\mathrm{C} 4-\mathrm{C} 5-\mathrm{H} 5$ & -177.8 & -179.4 & -180 & 180 & 179.0 & -179.6 \\
\hline $\mathrm{C} 3-\mathrm{C} 4-\mathrm{C} 5-\mathrm{C} 6$ & $2(1)$ & $0.6(7)$ & $-0(2)$ & $0(2)$ & $-1.1(9)$ & $0(1)$ \\
\hline $\mathrm{H} 4-\mathrm{C} 4-\mathrm{C} 5-\mathrm{H} 5$ & 2 & 0.6 & 0 & -0 & -1 & 0 \\
\hline $\mathrm{H} 4-\mathrm{C} 4-\mathrm{C} 5-\mathrm{C} 6$ & -177.8 & -179.4 & 180 & -180 & 179.0 & -179.7 \\
\hline $\mathrm{C} 4-\mathrm{C} 5-\mathrm{C} 6-\mathrm{S} 1$ & $177.0(7)$ & $178.8(3)$ & $0(3)$ & $-180(1)$ & $-178.6(4)$ & $-178.9(6)$ \\
\hline $\mathrm{C} 4-\mathrm{C} 5-\mathrm{C} 6-\mathrm{C} 7$ & $-1(1)$ & $-0.8(6)$ & $176(1)$ & $-0(2)$ & $0.6(8)$ & $2(1)$ \\
\hline $\mathrm{H} 5-\mathrm{C} 5-\mathrm{C} 6-\mathrm{S} 1$ & -3 & -1.2 & -4 & 0 & 1.3 & 1 \\
\hline $\mathrm{H} 5-\mathrm{C} 5-\mathrm{C} 6-\mathrm{C} 7$ & 178.8 & 179.2 & 180 & -180 & -179.5 & -178.1 \\
\hline $\mathrm{S} 1-\mathrm{C} 6-\mathrm{C} 7-\mathrm{N} 1$ & $0.9(7)$ & $1.2(4)$ & $-2(2)$ & $-12(2)$ & $-0.4(5)$ & $-0.5(7)$ \\
\hline $\mathrm{S} 1-\mathrm{C} 6-\mathrm{C} 7-\mathrm{C} 2$ & $-178.3(5)$ & $-178.7(3)$ & $-177(2)$ & $180(1)$ & $-179.8(4)$ & $178.2(5)$ \\
\hline $\mathrm{C} 5-\mathrm{C} 6-\mathrm{C} 7-\mathrm{N} 1$ & $179.3(7)$ & $-179.1(3)$ & $175(2)$ & $168(2)$ & $-179.8(4)$ & $178.9(6)$ \\
\hline $\mathrm{C} 5-\mathrm{C} 6-\mathrm{C} 7-\mathrm{C} 2$ & $0(1)$ & $1.0(5)$ & $-0(3)$ & $0(3)$ & $0.8(7)$ & $-2.4(9)$ \\
\hline C9-C10-C11-F1 & $-9(2)$ & $-1.3(5)$ & $-2(2)$ & $-1(2)$ & $1.0(6)$ & $-8(1)$ \\
\hline C9-C10-C11-C12 & $173.9(9)$ & $178.9(3)$ & $-178(1)$ & $178(1)$ & $-179.0(5)$ & $-178.6(6)$ \\
\hline $\mathrm{C} 15-\mathrm{C} 10-\mathrm{C} 11-\mathrm{F} 1$ & $174(2)$ & $177.5(3)$ & $176(2)$ & $-179(1)$ & $178.7(4)$ & $172(1)$ \\
\hline $\mathrm{C} 15-\mathrm{C} 10-\mathrm{C} 11-\mathrm{C} 12$ & $-4(1)$ & $-2.2(5)$ & $-0(2)$ & $178(1)$ & $-1.3(8)$ & $1.1(9)$ \\
\hline C9-C10-C15-C14 & $-176.0(8)$ & $178.9(3)$ & $178(1)$ & $-178(1)$ & $179.0(5)$ & $-179.8(6)$ \\
\hline C9-C10-C15-H15 & 4 & -1.1 & -2 & 2 & -1.1 & 0.2 \\
\hline $\mathrm{C} 11-\mathrm{C} 10-\mathrm{C} 15-\mathrm{C} 14$ & $2(1)$ & $0.1(5)$ & $0(2)$ & $0(2)$ & $1.3(7)$ & $0.5(9)$ \\
\hline
\end{tabular}




\begin{tabular}{lllllll}
\hline $\mathrm{C} 11-\mathrm{C} 10-\mathrm{C} 15-\mathrm{H} 15$ & -178.3 & -179.9 & -180 & -180 & -178.8 & -179.6 \\
\hline
\end{tabular}

Table S6. Intermolecular hydrogen bonds in Form I, III, IV, V and VI.

\begin{tabular}{|c|c|c|c|c|c|}
\hline & $\mathrm{D}-\mathrm{H} \cdots \mathrm{A}$ & $\mathrm{d}(\mathrm{D}-\mathrm{H})$ & $\mathrm{d}(\mathrm{H} \cdots \mathrm{A})$ & $\mathrm{d}(\mathrm{D} \cdots \mathrm{A})$ & $<(\mathrm{DHA})$ \\
\hline \multirow[t]{4}{*}{ Form I } & $\mathrm{C}(16)-\mathrm{H}(16 \mathrm{~A}) \cdots \mathrm{S}(1)^{\# 1}$ & 0.97 & 2.98 & $3.86(2)$ & 150.2 \\
\hline & $\mathrm{C}(18)-\mathrm{H}(18 \mathrm{C}) \cdots \mathrm{O}(1)$ & 0.97 & 2.38 & $2.92(2)$ & 114.3 \\
\hline & $\mathrm{N}(2)-\mathrm{H}(2) \cdots \mathrm{O}(1)^{\# 1}$ & 0.86 & 2.09 & $2.855(6)$ & 148.0 \\
\hline & $\mathrm{C}(5)-\mathrm{H}(5) \cdots \mathrm{F}(1)^{\# 2}$ & 0.93 & 2.48 & $3.22(5)$ & 135.9 \\
\hline \multirow[t]{4}{*}{ Form III } & $\mathrm{C}(19)-\mathrm{H}(19 \mathrm{~A}) \cdots \mathrm{S}(1)^{\# 3}$ & 0.96 & 2.55 & $3.44(3)$ & 153.8 \\
\hline & $\mathrm{C}\left(16-\mathrm{H}(16 \mathrm{~B}) \cdots \mathrm{S}(1)^{\# 4}\right.$ & 0.97 & 2.94 & $3.87(3)$ & 159.1 \\
\hline & $\mathrm{N}(2)-\mathrm{H}(2) \cdots \mathrm{O}(1)^{\# 4}$ & 0.86 & 2.08 & $2.840(3)$ & 146.4 \\
\hline & $\mathrm{C}(5)-\mathrm{H}(5) \cdots \mathrm{O}(2)^{\# 5}$ & 0.93 & 2.55 & $3.43(3)$ & 157.0 \\
\hline \multirow[t]{5}{*}{ Form IV } & $\mathrm{C}(16)-\mathrm{H}(16 \mathrm{~A}) \cdots \mathrm{O}\left(1^{\mathrm{a}}\right)$ & 0.97 & 2.46 & $2.95(2)$ & 111.4 \\
\hline & $\mathrm{C}(12)-\mathrm{H}(12) \cdots \mathrm{S}(1)^{\# 6}$ & 0.93 & 3.02 & $3.902(13)$ & 158.2 \\
\hline & $\mathrm{C}(35)-\mathrm{H}(35 \mathrm{~B}) \cdots \mathrm{O}(4)$ & 0.97 & 2.37 & $2.88(2)$ & 112.2 \\
\hline & $\mathrm{N}(2)-\mathrm{H}(2) \cdots \mathrm{O}(1)^{\# 7}$ & 0.86 & 2.03 & $2.838(12)$ & 156.6 \\
\hline & $\mathrm{C}(15)-\mathrm{H}(15) \cdots \mathrm{F}(1)$ & 0.98 & 2.31 & $2.808(18)$ & 110.2 \\
\hline Form V & $\mathrm{N}(2)-\mathrm{H}(2) \cdots \mathrm{O}(1)^{\# 8}$ & 0.86 & 1.97 & $2.808(5)$ & 163.5 \\
\hline \multirow[t]{4}{*}{ Form VI } & $\mathrm{C}(18)-\mathrm{H}(18) \cdots \mathrm{O}\left(3^{\mathrm{a}}\right)^{\# 9}$ & 0.97 & 2.37 & $3.249(15)$ & 150.9 \\
\hline & $\mathrm{C}(19)-\mathrm{H}(19 \mathrm{~B}) \cdots \mathrm{F}(1)^{\# 9}$ & 0.96 & 2.29 & $3.10(4)$ & 141.6 \\
\hline & $\mathrm{N}(2)-\mathrm{H}(2) \cdots \mathrm{O}\left(1^{\mathrm{a}}\right)^{\# 10}$ & 0.86 & 1.93 & $2.740(8)$ & 160.6 \\
\hline & $\mathrm{C}(5)-\mathrm{H}(5) \cdots \mathrm{F}(1)^{\# 11}$ & 0.93 & 2.51 & $3.42(2)$ & 167.3 \\
\hline
\end{tabular}

${ }^{\# 1}$ Symmetry code for acceptor atom: $(-x+1,-y+2, z+0) .{ }^{\# 2}$ Symmetry code for acceptor atom: $\left(-\mathrm{y}+1,-\mathrm{x}+3 / 2, \mathrm{z}^{-1 / 4}\right)$. ${ }^{\# 3}$ Symmetry code for acceptor atom: $\left(\mathrm{x},-\mathrm{y}+1, \mathrm{z}^{+1 / 2}\right)$. ${ }^{\# 4}$ Symmetry code for acceptor atom: $\left(-\mathrm{x}+1, \mathrm{y},-\mathrm{z}^{+3 / 2}\right) .{ }^{\# 5}$ Symmetry code for acceptor atom: $\left(x,-y+2, z^{-1 / 2}\right)$. ${ }^{\# 6}$ Symmetry code for acceptor atom: $(-x,-y+1,-z+2)$. ${ }^{\# 7}$ Symmetry code for acceptor atom: $(-x+1,-y+2,-z+1) .{ }^{\# 8}$ Symmetry code for acceptor 
atom: $(-\mathrm{x}+1,-\mathrm{y}+2,-\mathrm{z}){ }^{\# 9}$ Symmetry code for acceptor atom: $(-\mathrm{x}+1,-\mathrm{y}+1,-\mathrm{z}+1)$. ${ }^{\# 10}$ Symmetry code for acceptor atom: $(-x+2,-y+1,-z+1)$. ${ }^{\# 11}$ Symmetry code for acceptor atom: $(\mathrm{x}, \mathrm{y}-1, \mathrm{z})$.

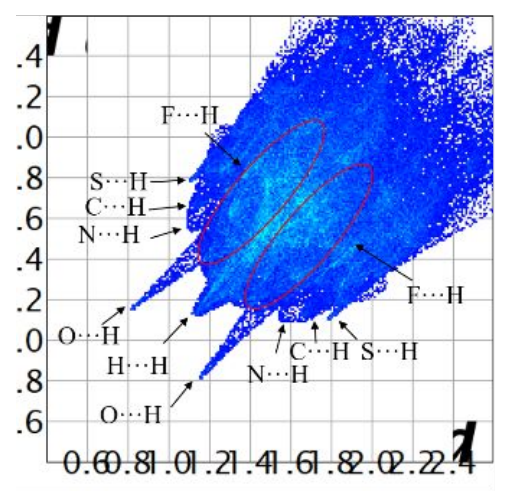

(a)

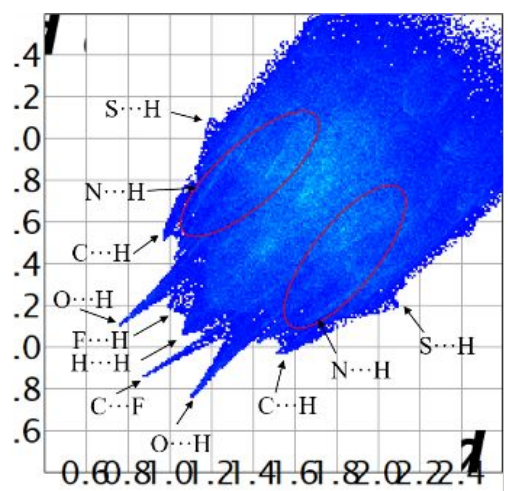

(c)

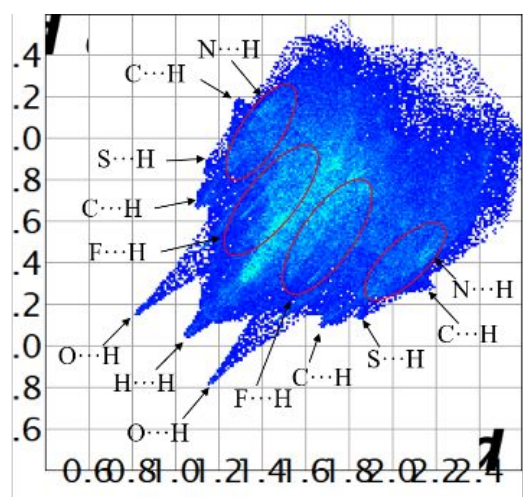

(b)

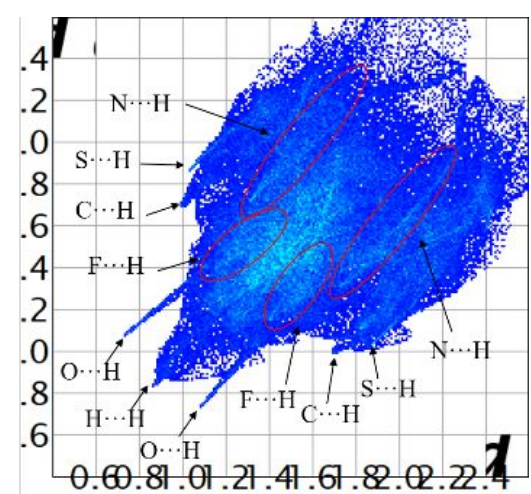

(d)

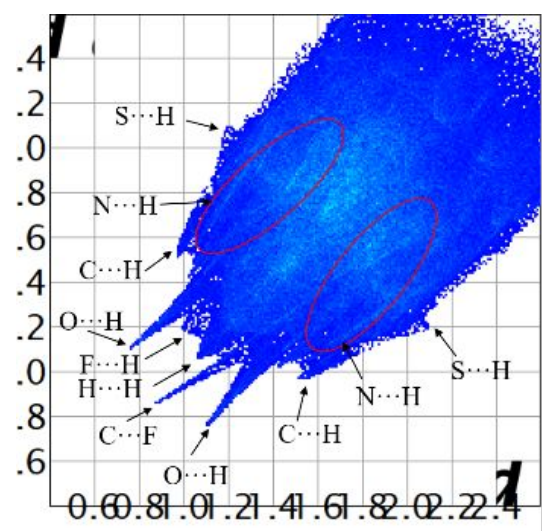

(e)

Figure S12. Two-dimensional fingerprint plots for DFL Forms: (a) I, (b) III, (c) IV, (d) 
V and (e) VI.

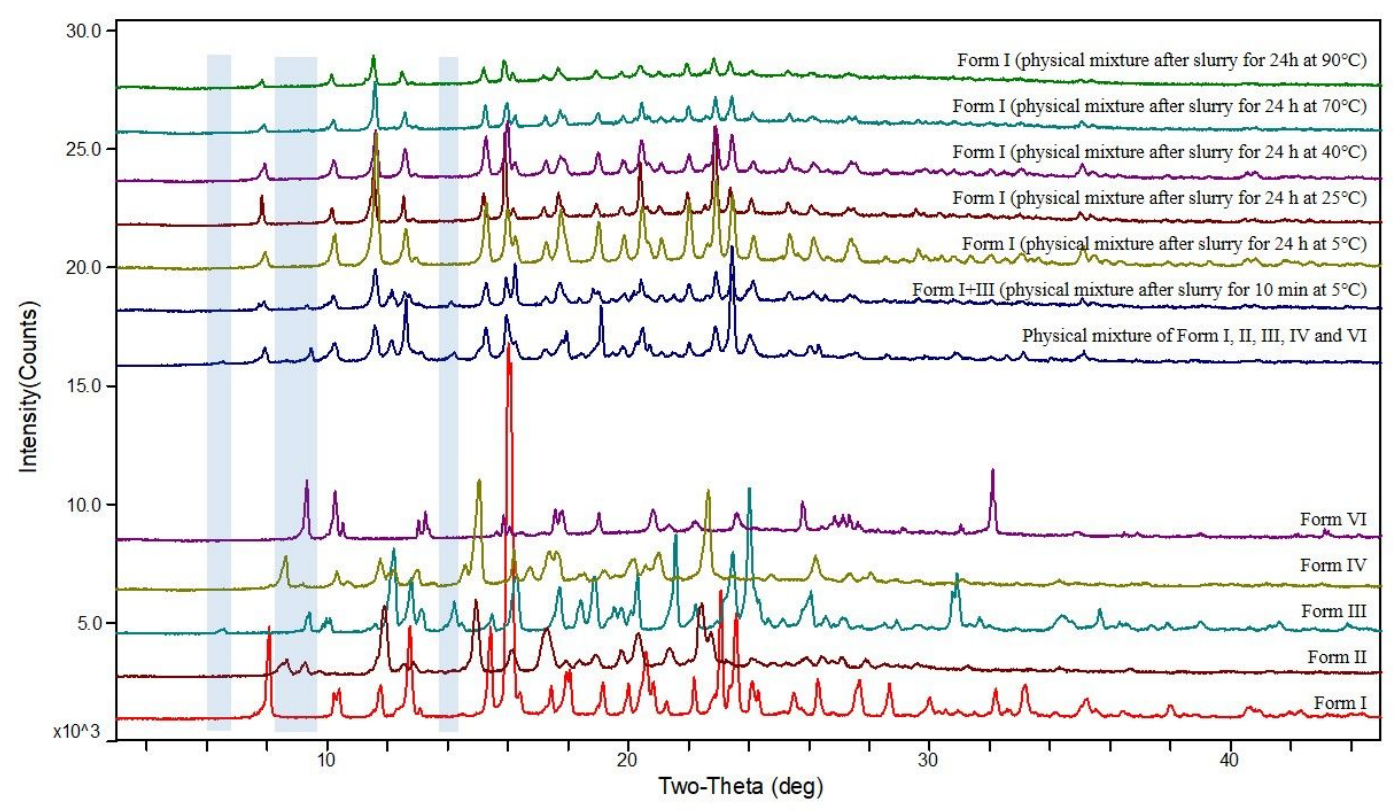

(a)

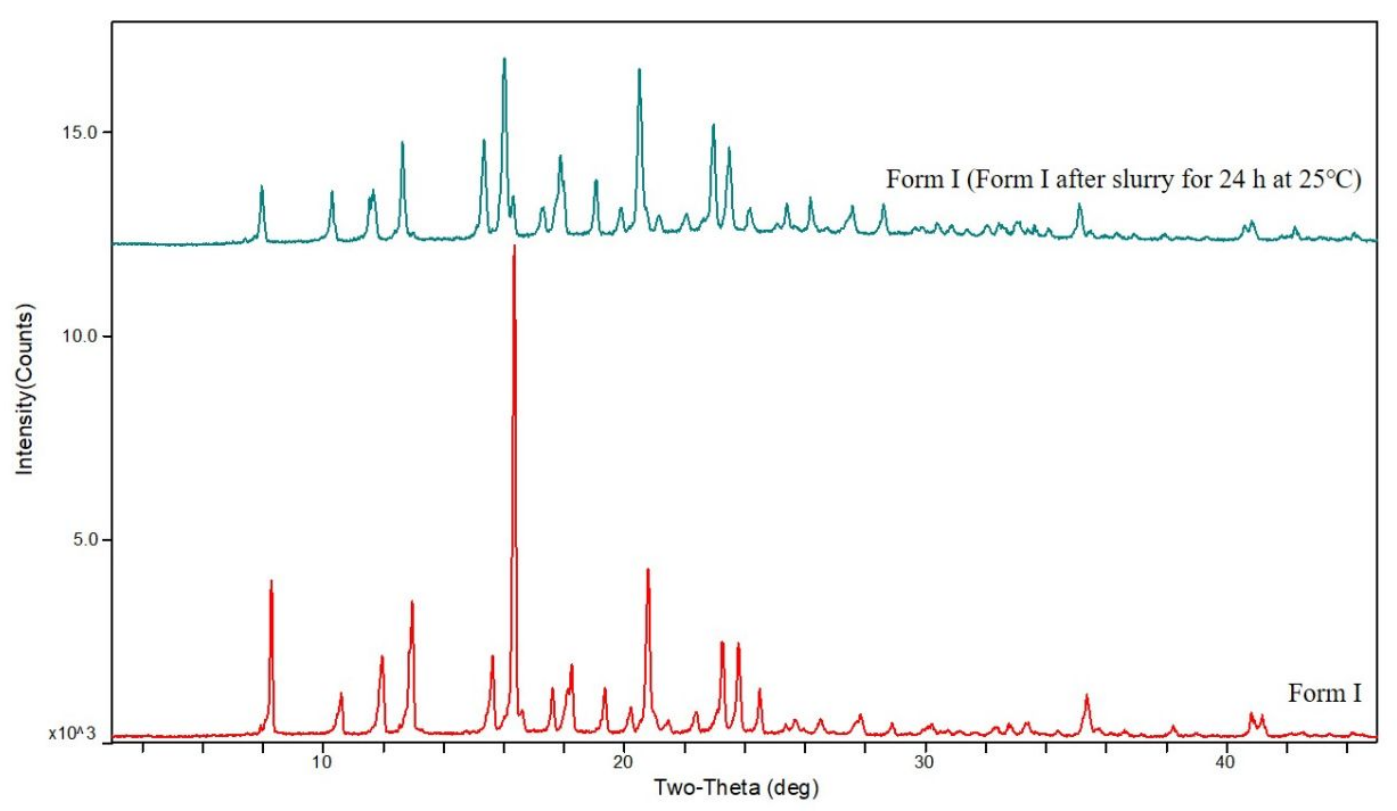

(b) 


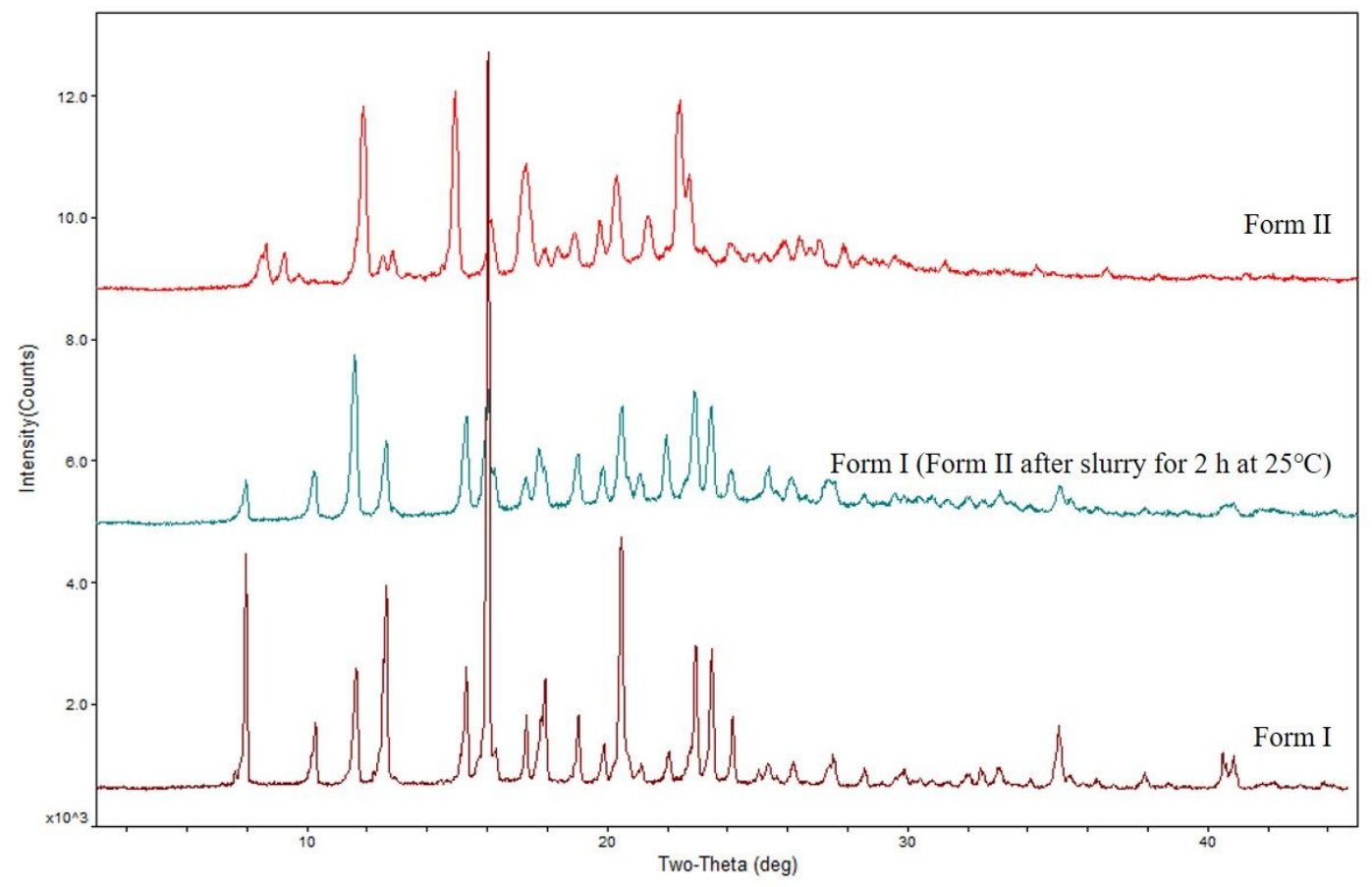

(c)

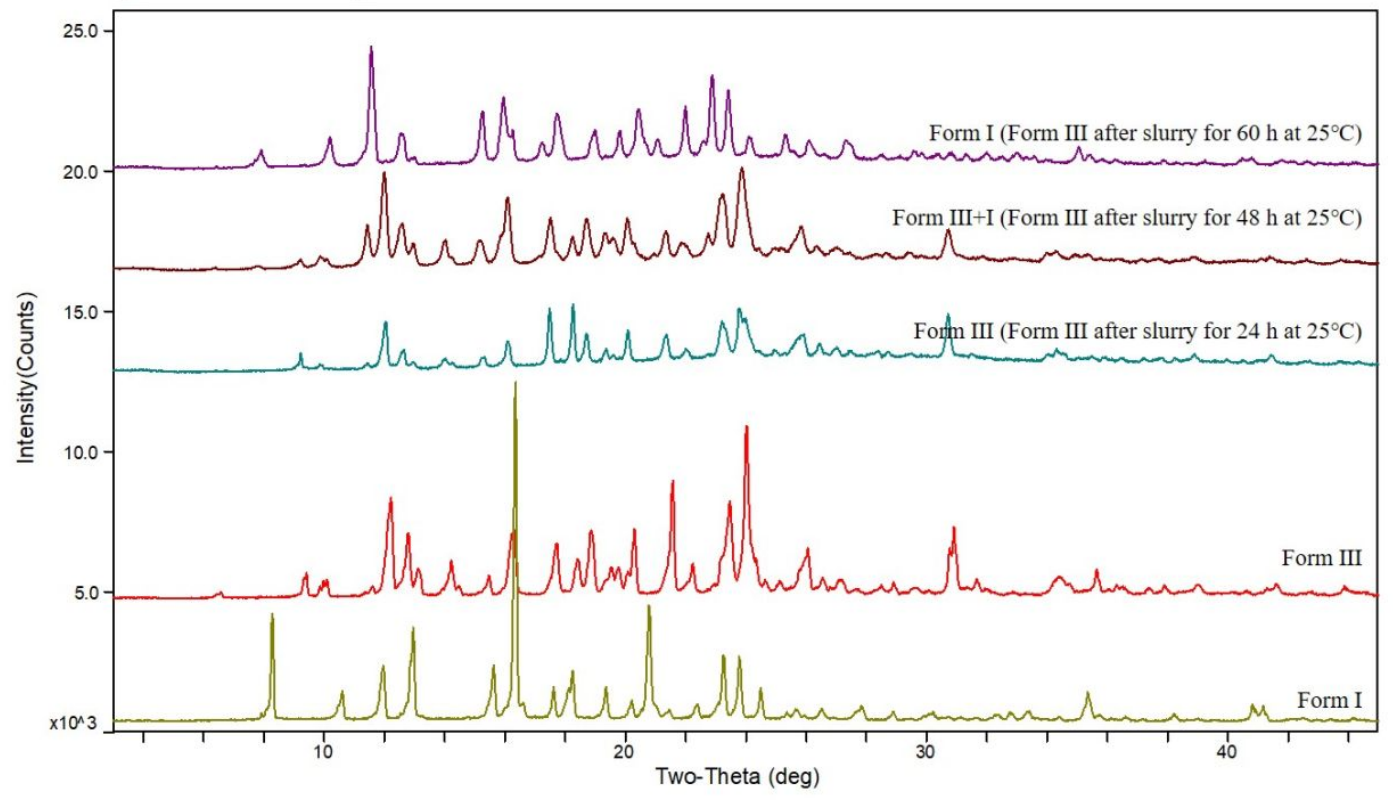

(d) 


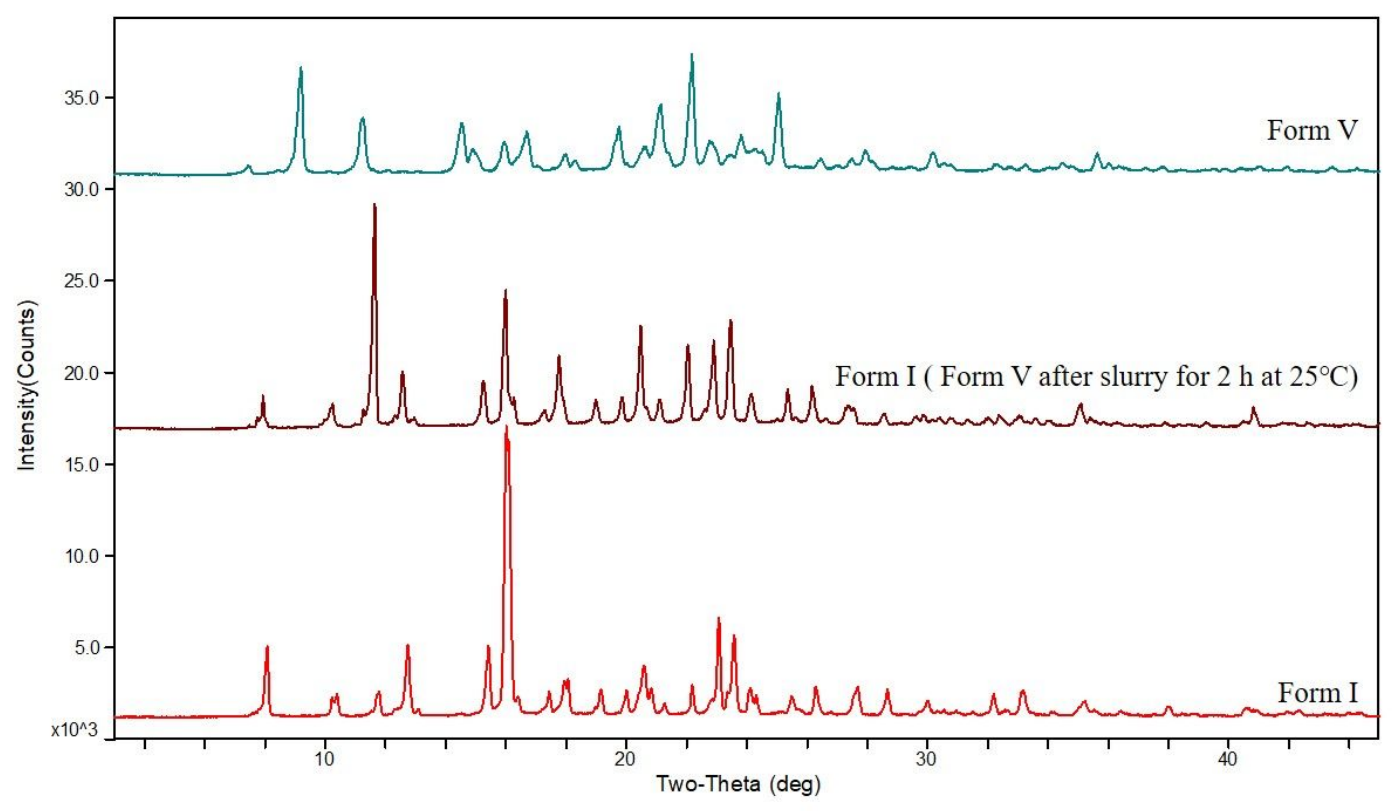

(e)

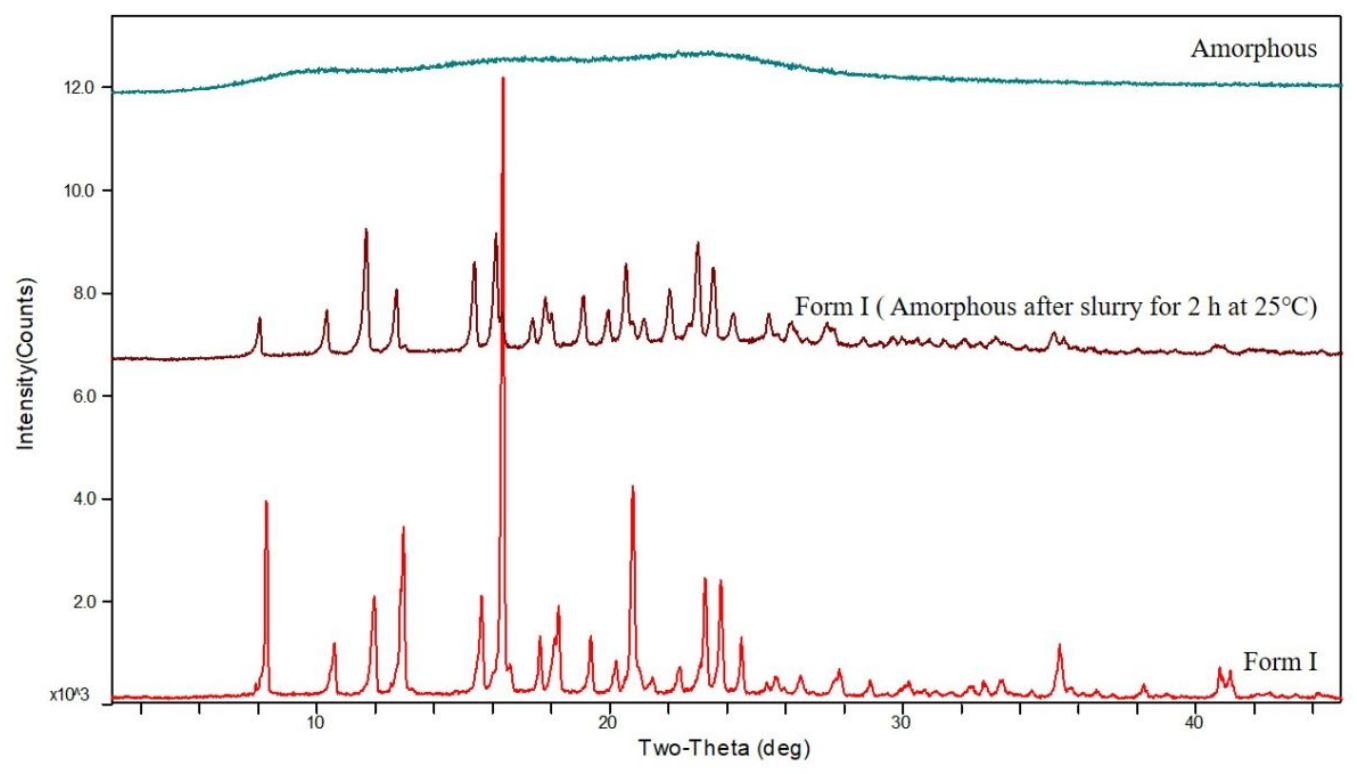

(f) 


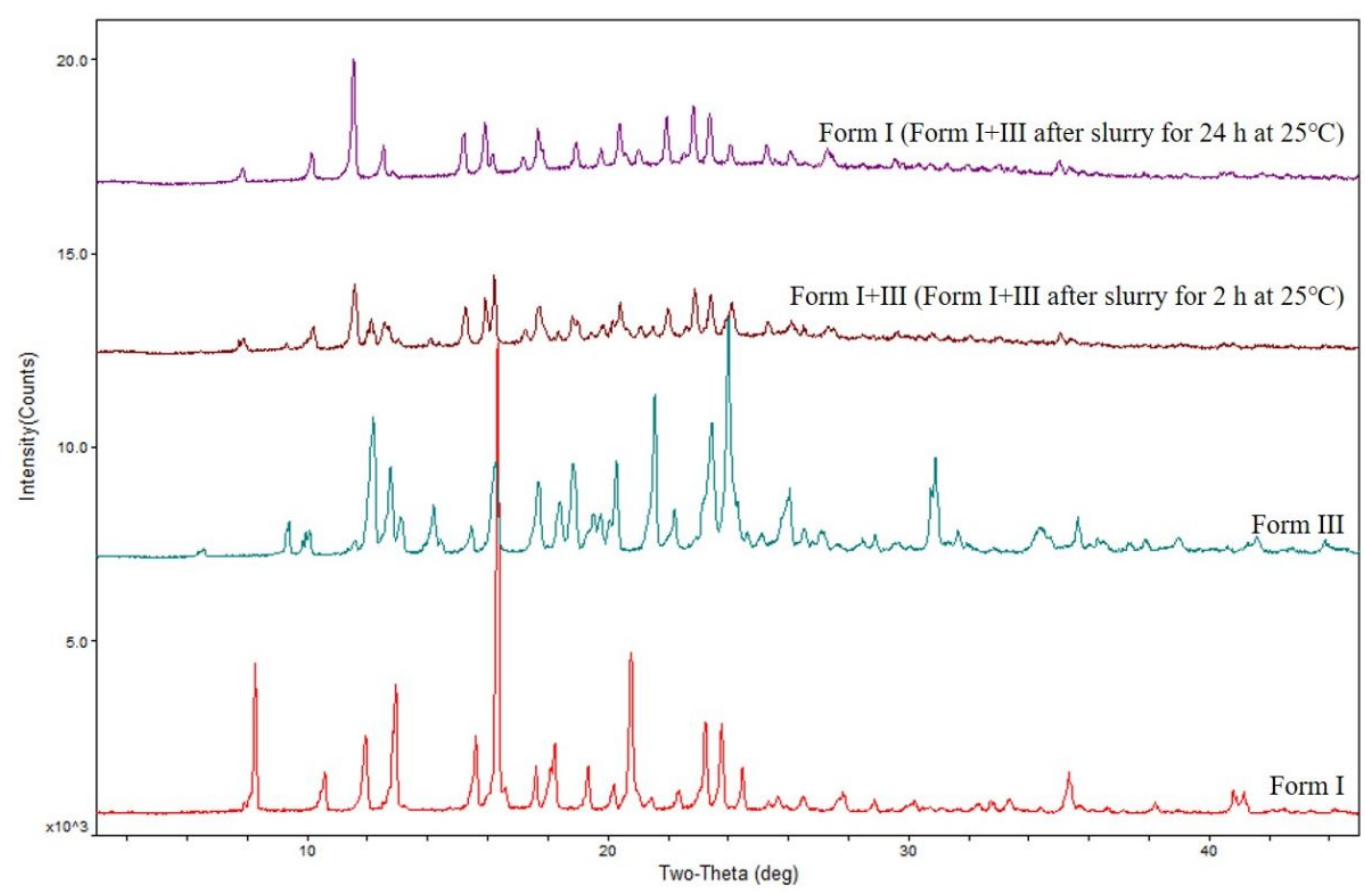

$(\mathrm{g})$

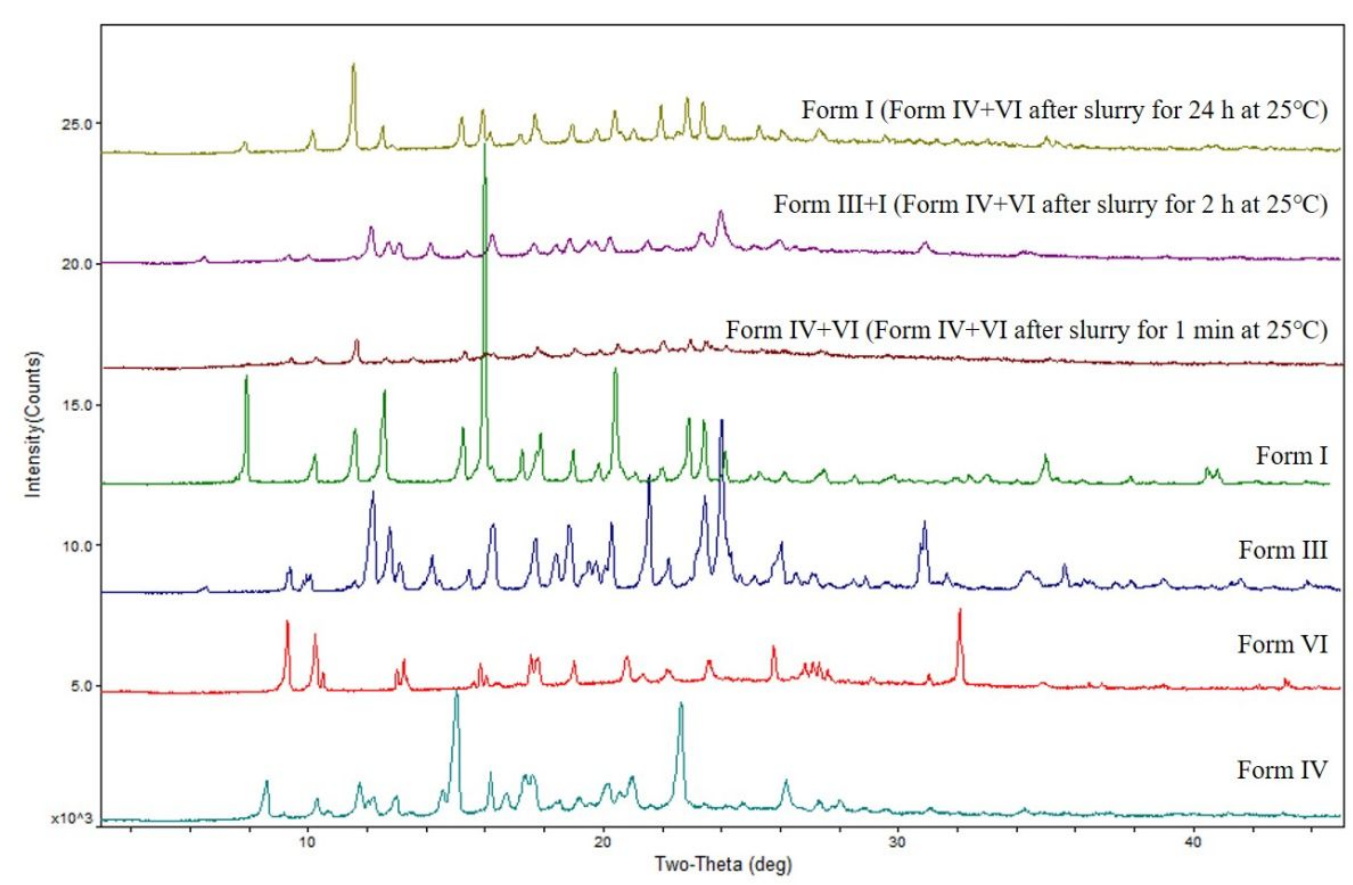

(h)

Figure S13. The PXRD patterns of DFL polymorphs in solvent-mediated suspension experiments. (a) The physically mixture of all DFL forms at different temperature, (b) Form I, (c) Form II, (d) Form III, (e) Form V, (f) amorphous, (g) the mixtures of Form 
I and III and (h) the mixtures of Form IV and VI.

Table S7. The design and results of solvent-mediated transformation experiments.

\begin{tabular}{|c|c|c|c|c|}
\hline Suspension solids & Solvents & Temperature & Time & Results \\
\hline & Ethanol & $5^{\circ} \mathrm{C}$ & $10 \mathrm{~min}$ & Form I+III \\
\hline & Ethanol & $5^{\circ} \mathrm{C}$ & $24 \mathrm{~h}$ & Form I \\
\hline Physical mixture of Form & Ethanol & $25^{\circ} \mathrm{C}$ & $24 \mathrm{~h}$ & Form I \\
\hline \multirow[t]{3}{*}{ I, II, III, IV and VI } & Ethanol & $40^{\circ} \mathrm{C}$ & $24 \mathrm{~h}$ & Form I \\
\hline & 1-butanol & $70^{\circ} \mathrm{C}$ & $24 \mathrm{~h}$ & Form I \\
\hline & 1-butanol & $90^{\circ} \mathrm{C}$ & $24 \mathrm{~h}$ & Form I \\
\hline Form I & Ethanol & $25^{\circ} \mathrm{C}$ & $24 \mathrm{~h}$ & Form I \\
\hline Form II & Ethanol & $25^{\circ} \mathrm{C}$ & $2 \mathrm{~h}$ & Form I \\
\hline Form III & Ethanol & $25^{\circ} \mathrm{C}$ & $24 \mathrm{~h}$ & Form III \\
\hline Form III & Ethanol & $25^{\circ} \mathrm{C}$ & $48 \mathrm{~h}$ & Form III+I \\
\hline Form III & Ethanol & $25^{\circ} \mathrm{C}$ & $60 \mathrm{~h}$ & Form I \\
\hline Form V & Ethanol & $25^{\circ} \mathrm{C}$ & $2 \mathrm{~h}$ & Form I \\
\hline Amorphous & Ethanol & $25^{\circ} \mathrm{C}$ & $2 \mathrm{~h}$ & Form I \\
\hline Physical mixture of Form I & Ethanol & $25^{\circ} \mathrm{C}$ & $2 \mathrm{~h}$ & Form I+III \\
\hline and III & Ethanol & $25^{\circ} \mathrm{C}$ & $24 \mathrm{~h}$ & Form I \\
\hline Physical mixture of Form & Ethanol & $25^{\circ} \mathrm{C}$ & $2 \mathrm{~h}$ & Form III+I \\
\hline IV and VI & Ethanol & $25^{\circ} \mathrm{C}$ & $24 \mathrm{~h}$ & Form I \\
\hline
\end{tabular}




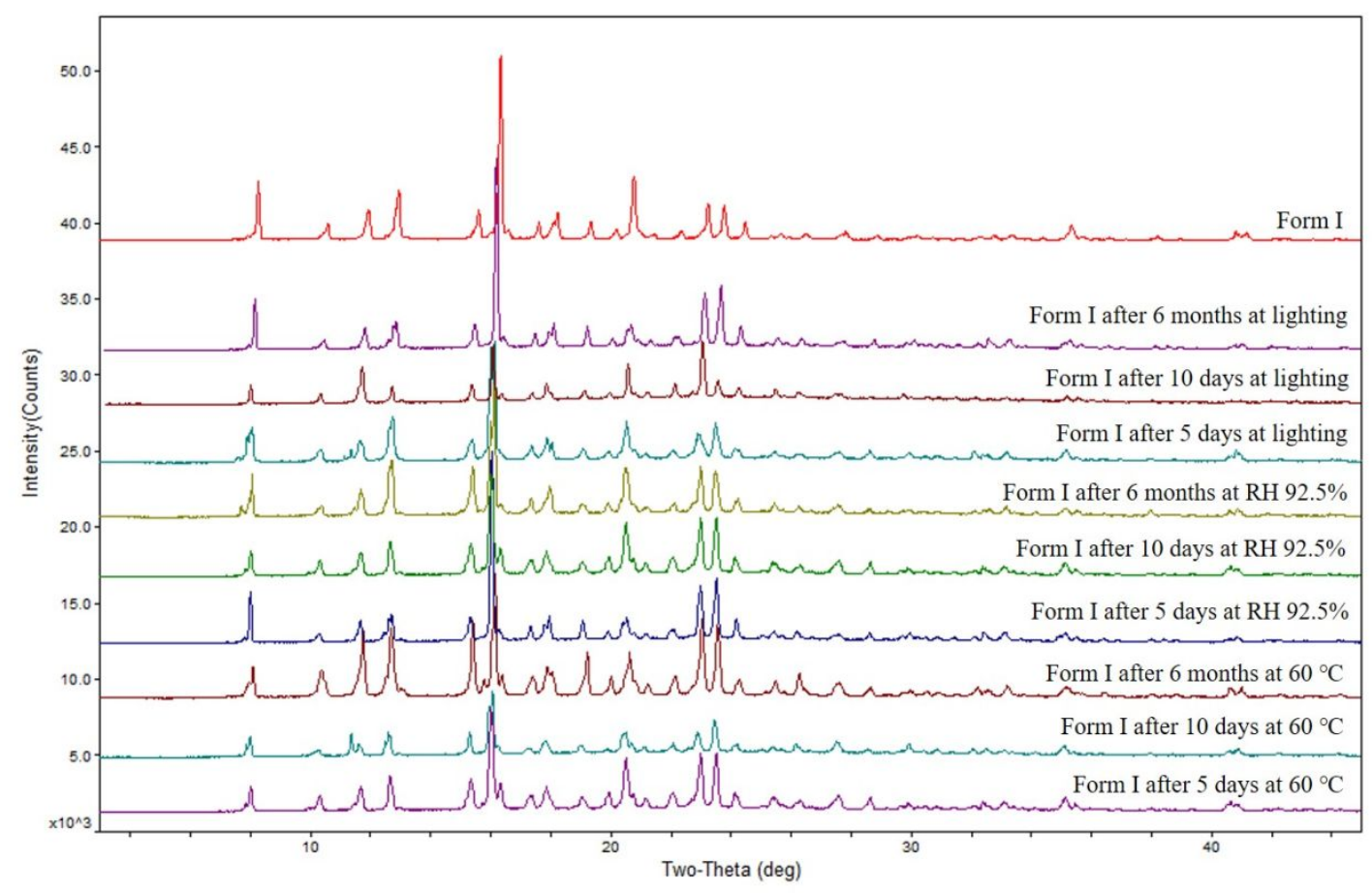

(a)

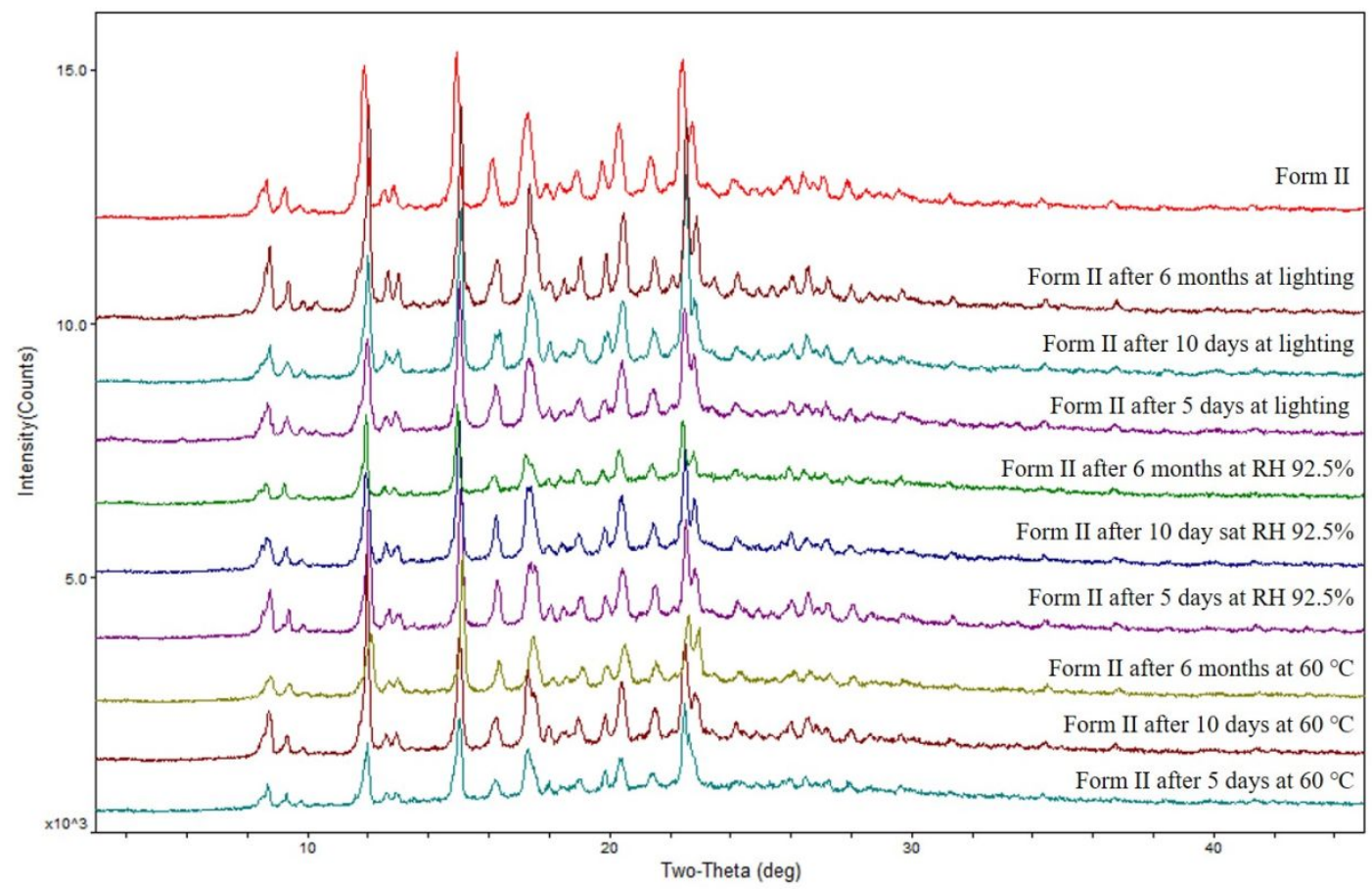

(b) 


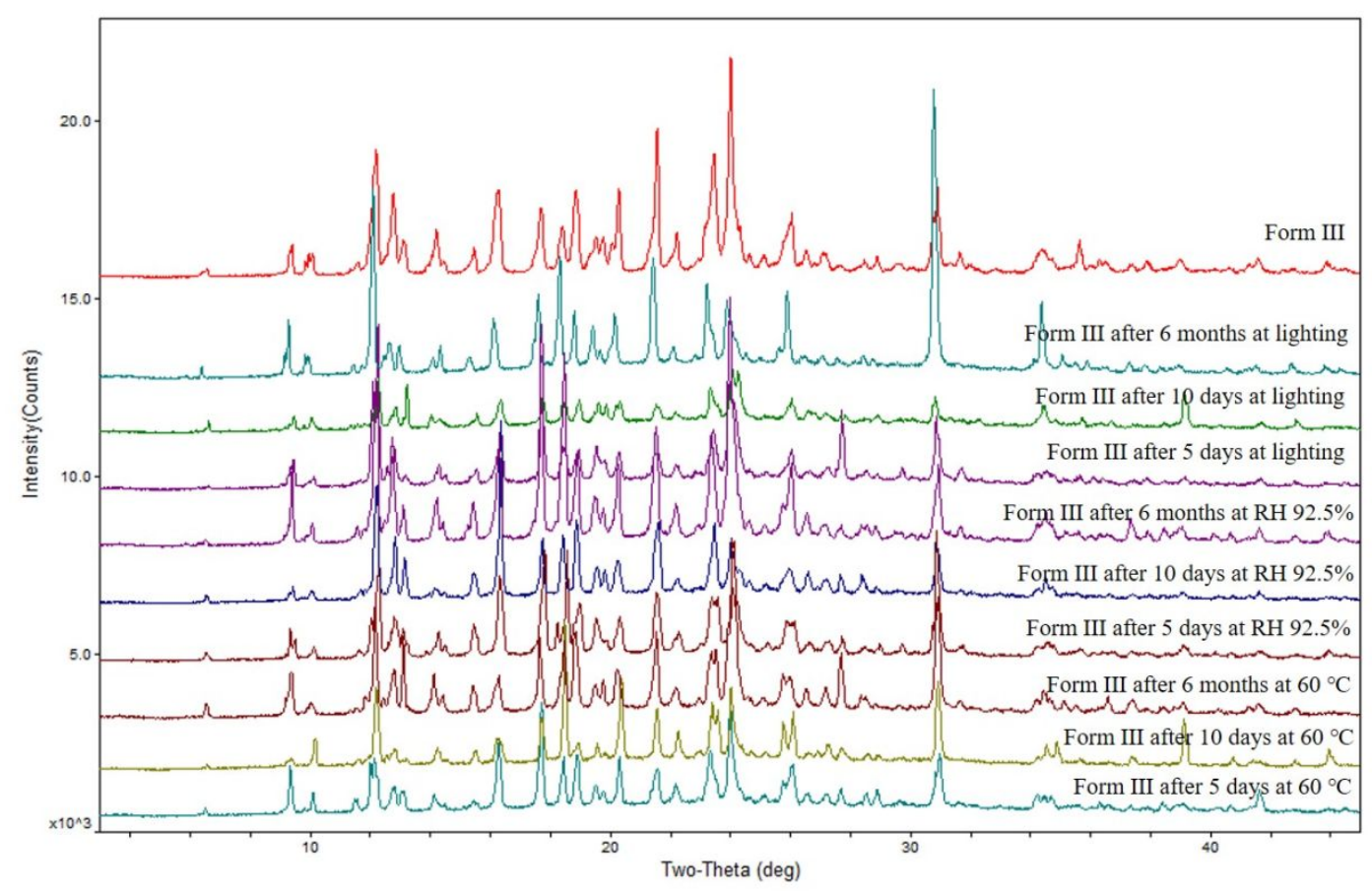

(c)

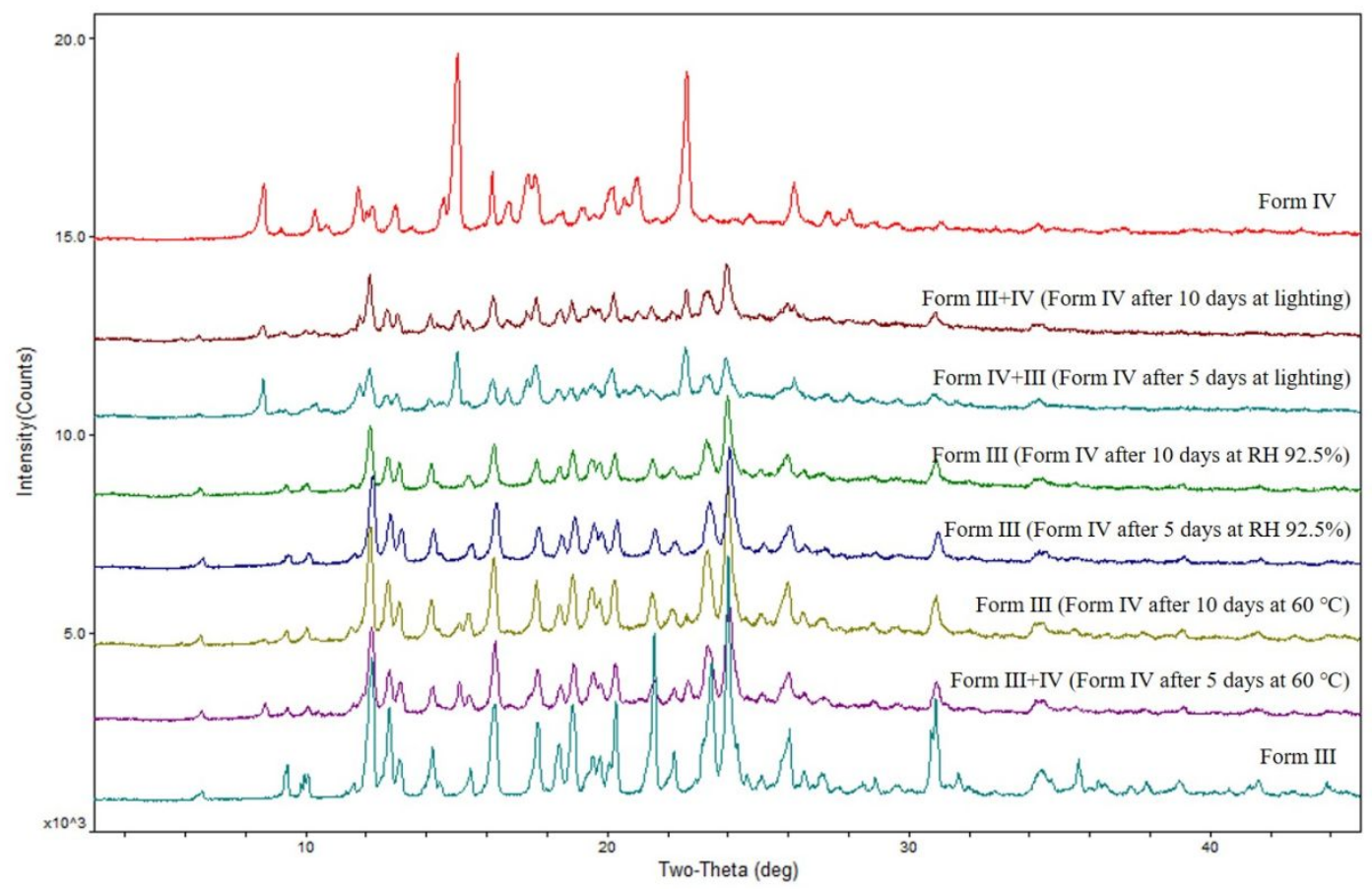

(d) 


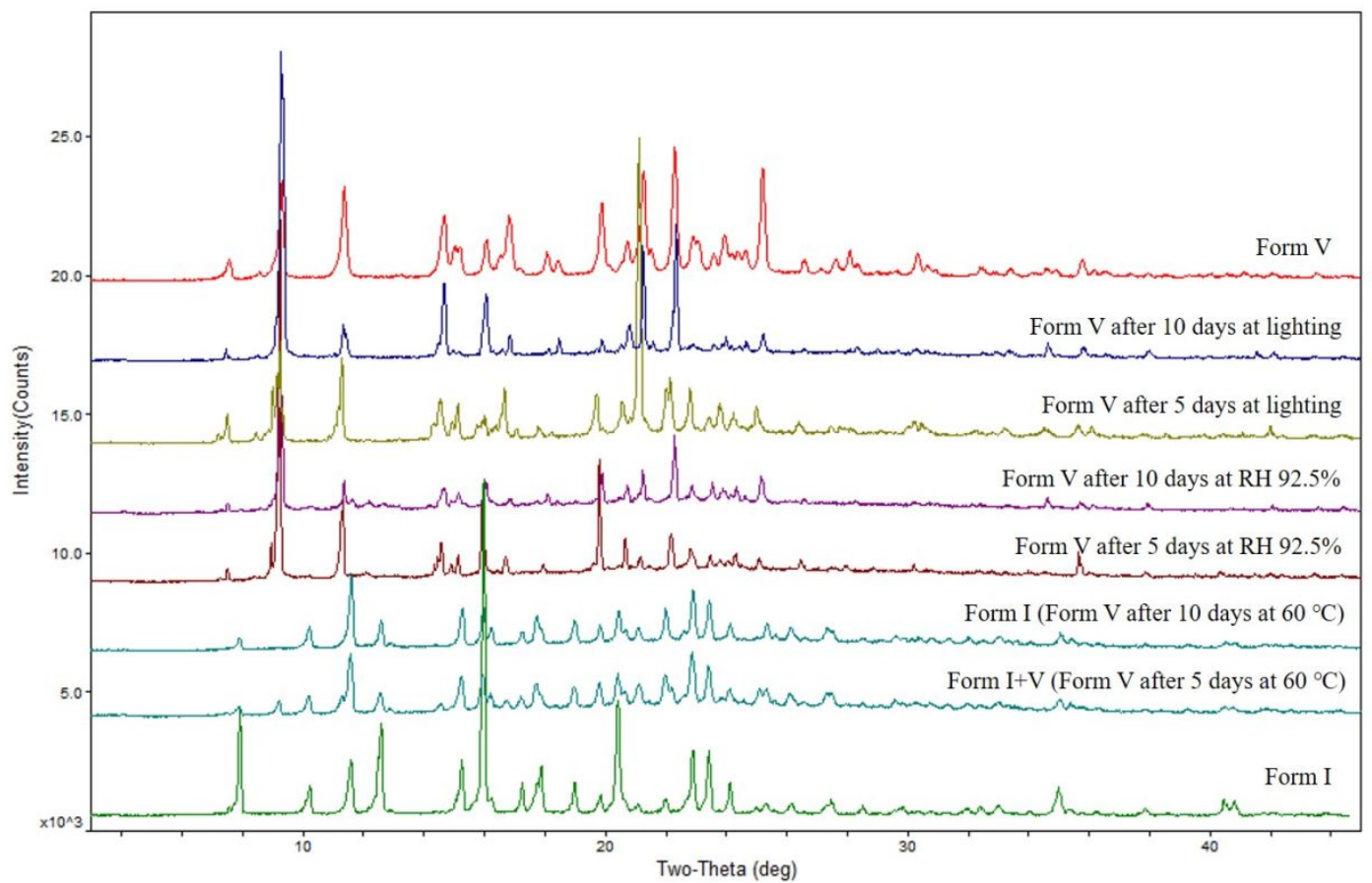

(e)

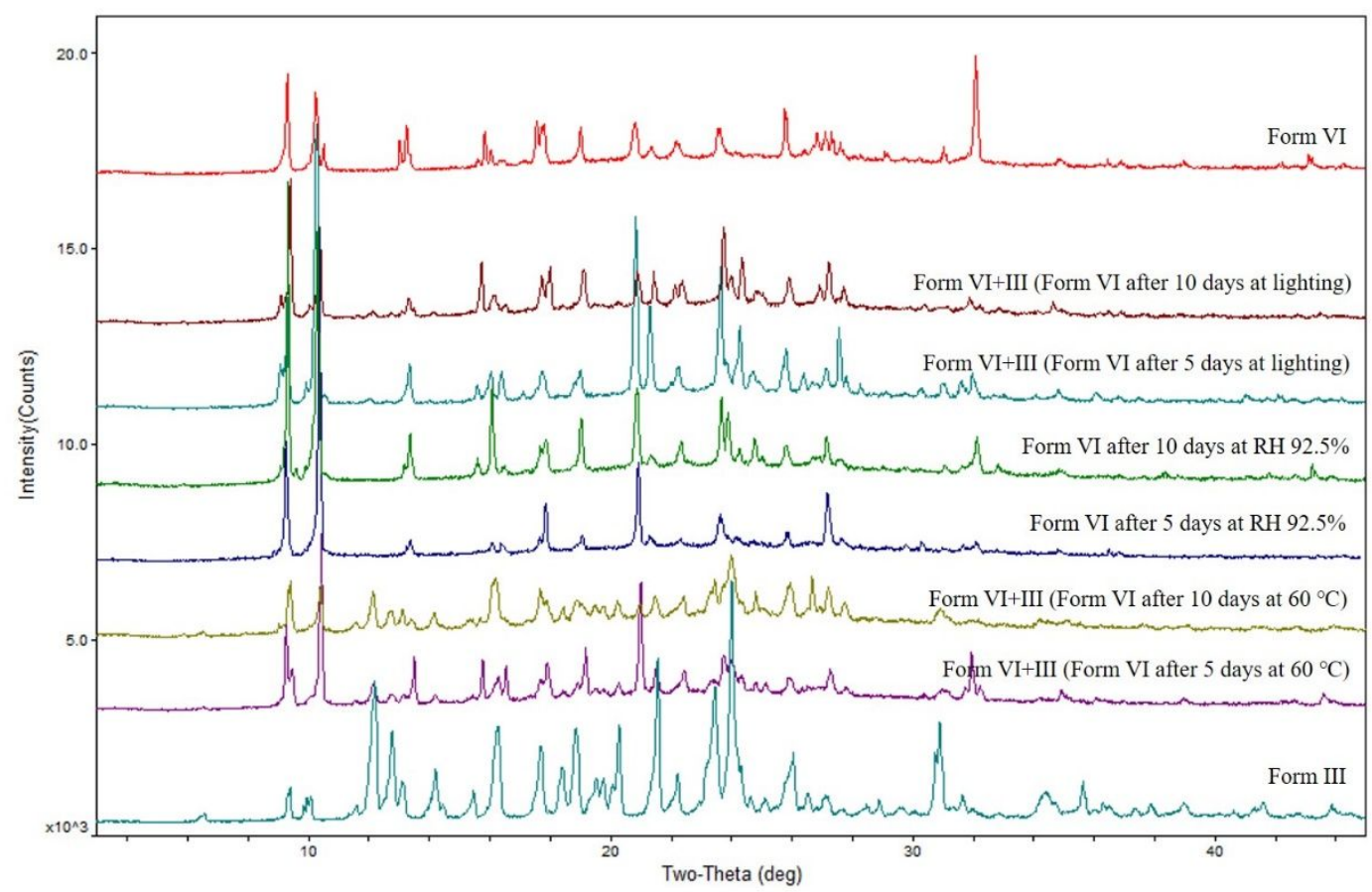

(f) 


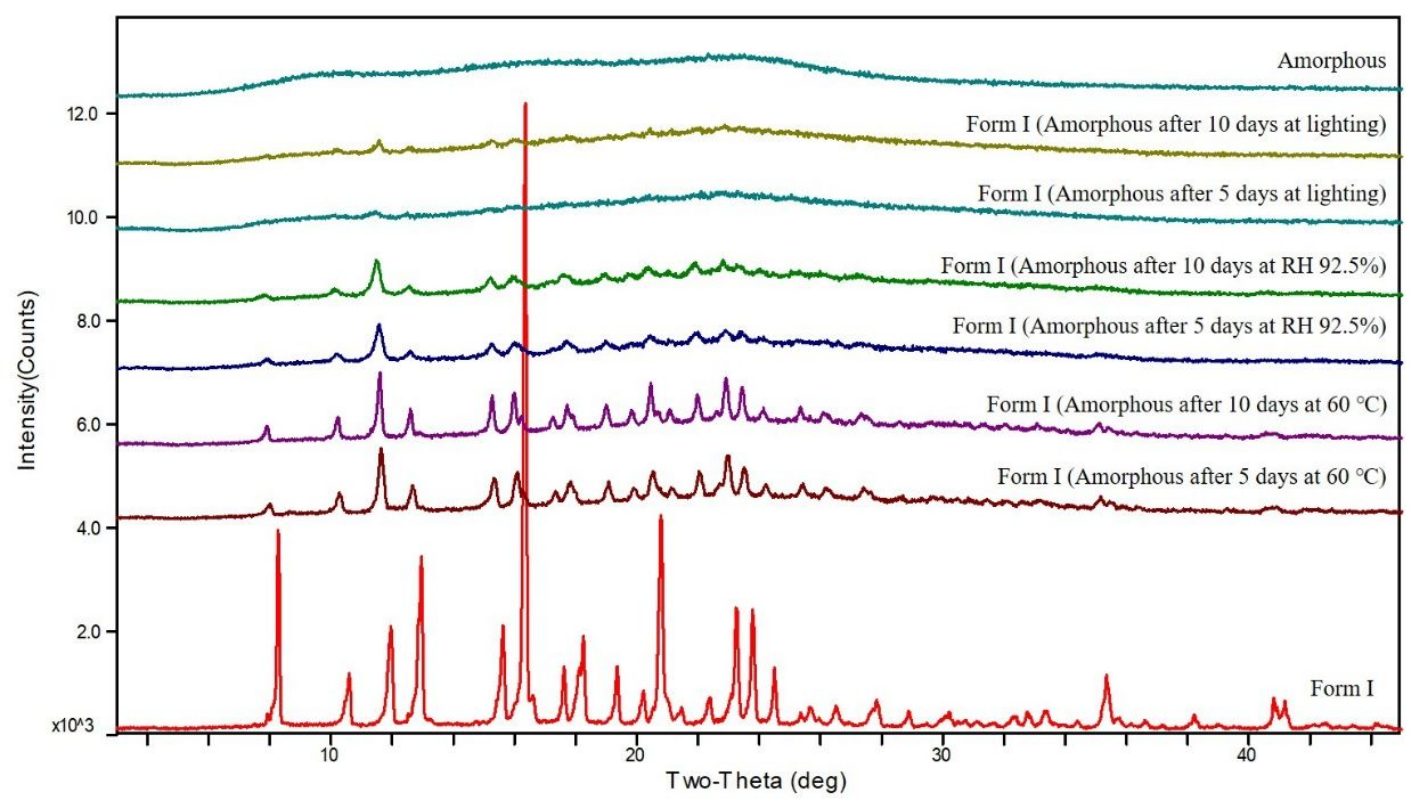

(g)

Figure S14. The PXRD patterns of DFL polymorphs in stability experiment. (a) Form I, (b) Form II, (c) Form III, (d) Form IV, (e) Form V, (f) Form VI, and (g) amorphous.

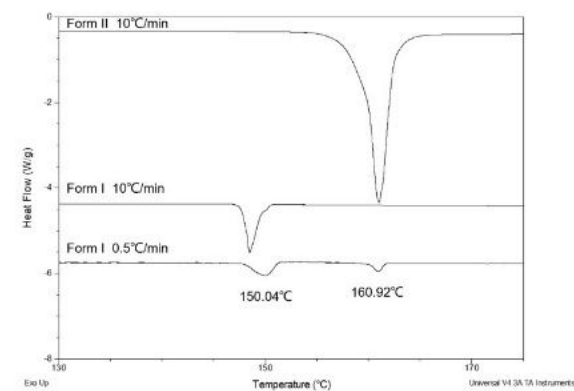

(a)

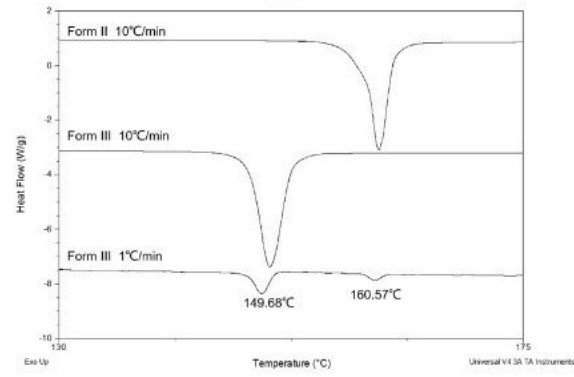

(c)

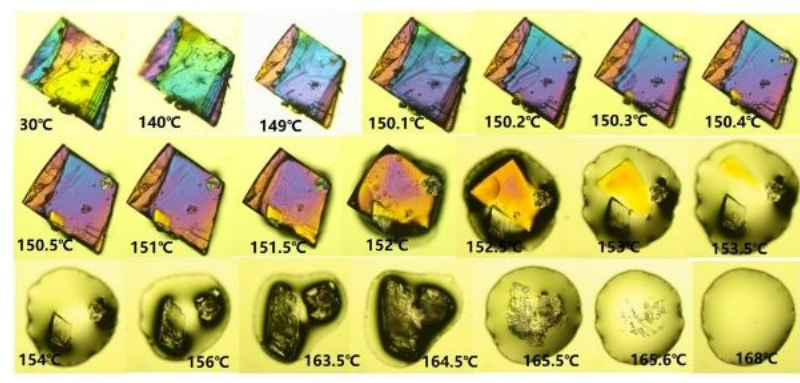

(b)

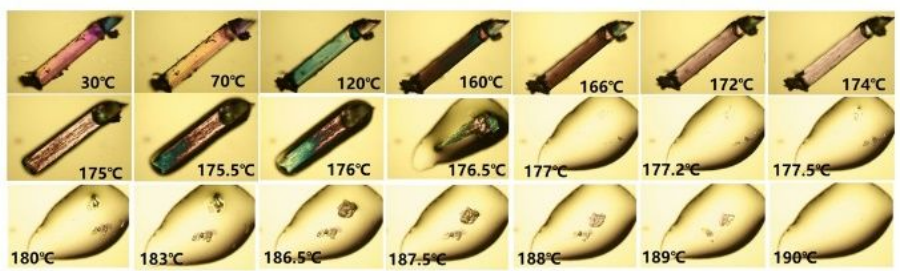

(d)

Figure S15. (a) Overlay of DSC curves of Form I at different heating rates, (b) 
Polarized-light photographs show the selected temperature-induced solid-state transformations of DFL (I $\rightarrow$ II), (c) Overlay of DSC curves of Form III at different heating rates, and (d) Polarized-light photographs show the selected temperature induced solid-state transformations of DFL (III $\rightarrow$ II).

Table S8. Solubility data of DFL Forms I and III at different temperatures.

\begin{tabular}{|c|c|c|}
\hline \multirow{2}{*}{$\mathrm{T}\left({ }^{\circ} \mathrm{C}\right)$} & \multicolumn{2}{|c|}{ Solubility $\left(\mathrm{mg} \cdot \mathrm{mL}^{-1}\right)$} \\
\hline & Form I & Form III \\
\hline 5 & 8.98 & 11.53 \\
\hline 7.5 & 9.73 & 13.04 \\
\hline 10 & 10.60 & 14.85 \\
\hline 12.5 & 11.80 & 16.84 \\
\hline 15 & 12.79 & 18.99 \\
\hline 17.5 & 14.00 & 21.22 \\
\hline 20 & 15.18 & 23.35 \\
\hline 22.5 & 16.38 & 26.05 \\
\hline 25 & 19.15 & 29.01 \\
\hline 30 & 23.52 & 36.10 \\
\hline 35 & 29.78 & 43.03 \\
\hline 40 & 37.24 & 60.35 \\
\hline 45 & 47.53 & 81.45 \\
\hline
\end{tabular}




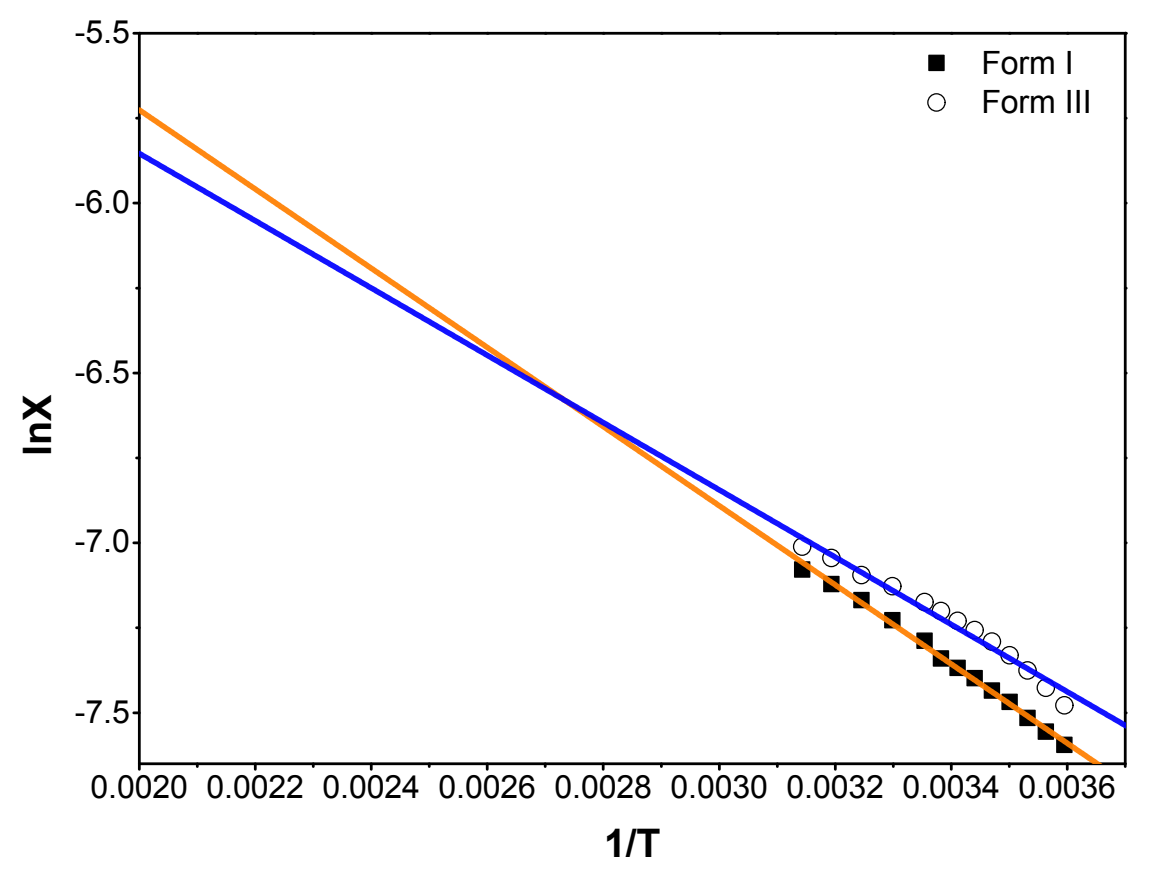

Figure S16. Van't Hoff plots of log solubility for DFL Forms I and III in ethanol.

Table S9. Fitting parameters of Van't Hoff equation for DFL Form I and III.

\begin{tabular}{cccc}
\hline & Intercept & Slope & $\mathrm{R}^{2}$ \\
\hline Form I & -1162.6 & -3.4040 & 0.9964 \\
Form III & -1001.9 & -3.8331 & 0.9764 \\
\hline
\end{tabular}




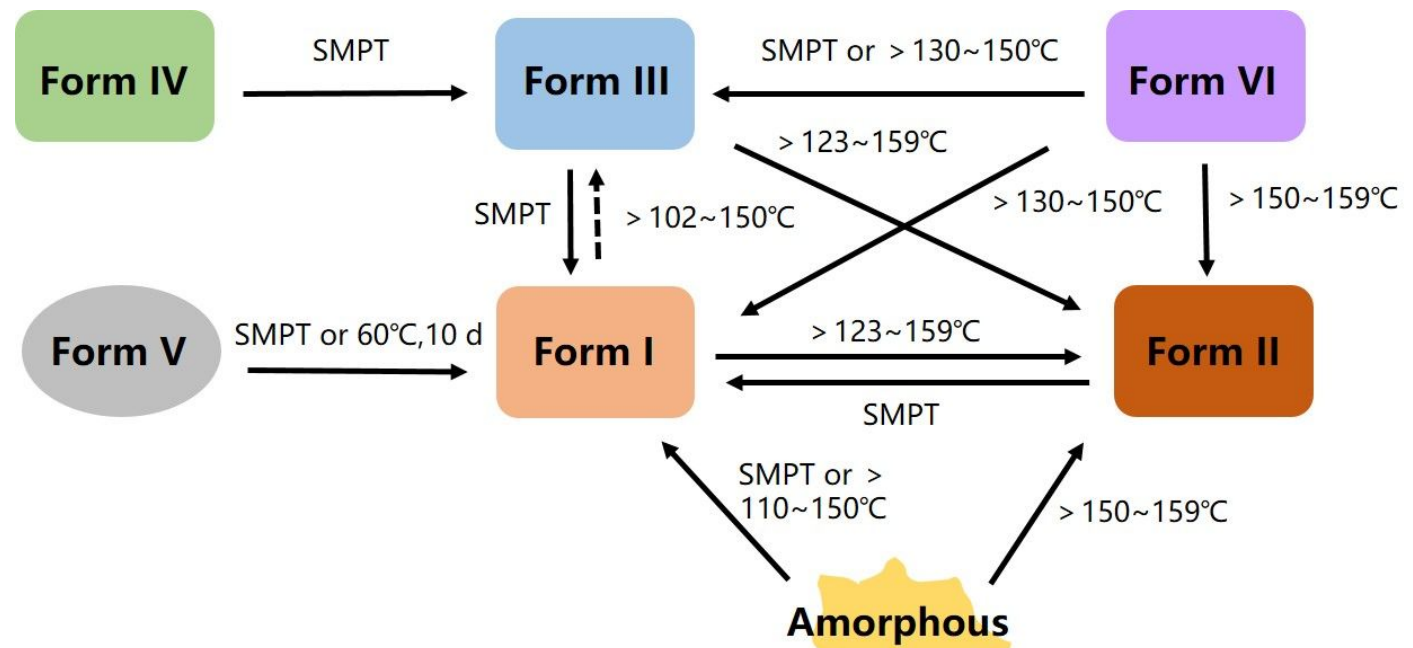

Figure S17. The transformation relationship of DFL polymorphs.

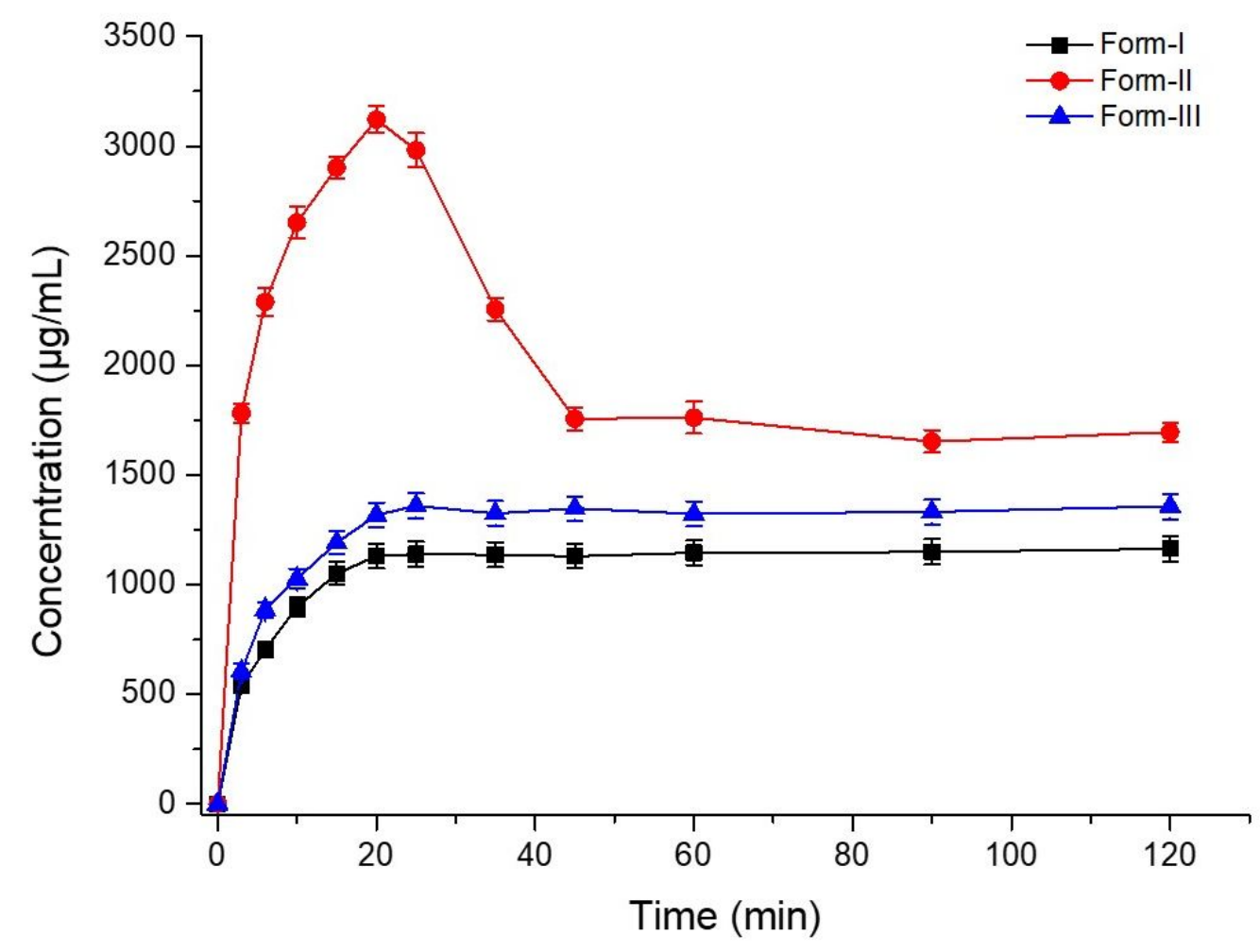

Figure S18. Dissolution-time profiles of DFL Forms I, II, and III in 50\% v/v ethanol at $25^{\circ} \mathrm{C}$. The results were expressed as mean $\pm \mathrm{SD}, \mathrm{n}=3$. 
